\title{
Pacific
}

Journal of

Mathematics

VERMA MODULES INDUCED FROM NONSTANDARD BOREL SUBALGEBRAS

BEN COX

Volume $165 \quad$ No. 2

October 1994 
VERMA MODULES INDUCED FROM NONSTANDARD BOREL SUBALGEBRAS

\section{BEN COX}

In this paper we study composition series and embeddings of Verma modules induced from "nonstandard" Borel subalgebras. This article can be viewed as a generalization of Futorny's work on imaginary Verma modules for $A_{1}^{(1)}$ where the center of the Kac-Moody algebra acts nontrivially.

Introduction. Let $A$ be an indecomposable symmetrizable generalized Cartan matrix, $\mathfrak{g}(A)=\mathfrak{n}_{-} \oplus \mathfrak{H} \oplus \mathfrak{n}_{+}$the triangular decomposition of the Kac-Moody algebra $\mathfrak{g}(A)$ and $W$ the Weyl group for $\mathfrak{g}(A)$. The "standard" Borel subalgebra $\mathfrak{b}_{+}$and its opposite $\mathfrak{b}_{-}$ are defined to be $\mathfrak{b}_{ \pm}=\mathfrak{h} \oplus \mathfrak{n}_{ \pm}$. For affine Kac-Moody algebras, H. Jakobsen and V. Kac and independently V. Futornyi have found an explicit description of a set of representatives of the conjugacy classes of Borel subalgebras (see 1.1) under the action of $W \times\{ \pm 1\}$. We will call all Borel subalgebras not conjugate to $\mathfrak{b}_{+}$or $\mathfrak{b}_{-}$, "nonstandard" Borel subalgebras. In particular for each subset $X$ of the set of simple roots $\Pi$ for $\mathfrak{g}(A)$, one can construct a nonstandard Borel subalgebra $\mathfrak{b}_{+}^{X}$ and then for each $\lambda \in \mathfrak{H}^{*}$ one can use induction to obtain what we will call a "nonstandard" Verma module $M^{X}(\lambda)$. For example if $X=\Pi$ then $\mathfrak{b}_{+}^{X}$ is the standard Borel subalgebra and $M(\lambda)$ is the "standard" Verma module. At the other extreme $X=\emptyset$ one obtains the "natural" Borel subalgebra and what one might call a "natural" Verma module. A striking difference between Verma modules induced from a standard Borel and those induced from $\mathfrak{b}_{+}^{X}$ for $X \subsetneq \Pi$, is that these new nonstandard Verma modules have infinite dimensional weight spaces. Consequently many of the classical techniques used in the study of the composition series of standard Verma modules do not seem to apply to this new setting. 
The purpose of this article is to describe, in the case that the center of $\mathfrak{g}(A)$ acts nontrivially, the composition series and embeddings of Verma modules induced from "nonstandard" Borel subalgebras. More precisely the main results appear as Theorem 5.2 and Corollary 5.3 where they assert the following: The multiplicity of irreducible modules in a local composition series for a Verma module, $M(\lambda)$, induced by a nonstandard Borel subalgebra $\mathfrak{b}_{+}$(see 1.1) is the same as the multiplicity of irreducibles in a local composition series for a Verma module, $M_{\mathfrak{k}}(\lambda)$, induced by a standard Borel subalgebra $\hat{\mathfrak{k}}_{+} \subset \mathfrak{b}_{+}$. Moreover there exists an isomorphism of vector spaces $\operatorname{Hom}_{\hat{\mathfrak{g}}}(M(\lambda), M(\mu)) \cong \operatorname{Hom}_{\hat{\mathfrak{k}}}\left(M_{\mathfrak{k}}(\lambda), M_{\mathfrak{k}}(\mu)\right)$ for $\mu \in \mathfrak{H}^{*}$. Unexpectedly the representation theory of infinite dimensional Heisenberg algebras plays an important role in the proof of these results (see the proofs of 2.3 , and 4.5).

This paper can be viewed as an extension of work done in [4] where Futorny studied composition series and embeddings of Verma modules for $\mathfrak{g}(A)=A_{1}^{(1)}$. See also [1] and [2] for work related to natural Verma modules.

The author would like to thank Thomas J. Enright for many helpful suggestions that improved the exposition of this article and also Nolan Wallach for simplifying the proof of Lemma 4.2.

\section{Notation.}

1.1. For any relation $R$ on the set of integers and $c$ an integer we set $\mathbb{Z}_{R c}=\{a \in \mathbb{Z} \mid a R c\}$. For example $\mathbb{Z}_{>0}$ is just the set of positive integers. Let $\dot{\mathfrak{g}}$ be a simple finite dimensional Lie algebra over $\mathbb{C}$, $\mathfrak{h}$ a Cartan subalgebra of $\dot{\mathfrak{g}}, \dot{\Delta}$ its root system with respect to $\mathfrak{h}$, and $I I$ a set of simple roots and $\dot{\Delta}_{+}\left(\right.$resp. $\left.\dot{\Delta}_{-}\right)$the set of positive (resp. negative) roots determined by $\Pi$. Let $\dot{\mathfrak{n}}_{ \pm}:=\bigoplus_{\alpha \in \dot{\Delta}_{ \pm}} \dot{\mathfrak{g}}_{\alpha}$ so that $\dot{\mathfrak{g}}=\mathfrak{n}_{-} \oplus \mathfrak{h} \oplus \mathfrak{n}_{+}$is the triangular decomposition of $\dot{\mathfrak{g}}$. For any Lie algebra $\mathfrak{a}$, let $L(\mathfrak{a})=\mathfrak{a} \otimes \mathbb{C}\left[t, t^{-1}\right]$ be the loop algebra of $\mathfrak{a}$ and let

$$
\hat{\mathfrak{g}}=L(\dot{\mathfrak{g}}) \oplus \mathbb{C} c \oplus \mathbb{C d}
$$

be the associated nontwisted affine Kac-Moody algebra of $\dot{\mathfrak{g}}$ (see [8] and [9] for more information about these algebras). We let

$$
\mathfrak{H}=\mathfrak{h}+\mathbb{C} c+\mathbb{C} d
$$

denote the Cartan subalgebra of $\hat{\mathfrak{g}}$. 
Let $\delta$ be the indivisible positive imaginary root for $\hat{\mathfrak{g}}$ and let $\Delta=$ $\{\alpha+n \delta \mid \alpha \in \dot{\Delta}, n \in \mathbb{Z}\}$ be the set of roots of $\hat{\mathfrak{g}}$. As in [7] we call a subset $\Delta_{+}$of $\Delta$ a set of positive roots if

(1) If $\alpha, \beta \in \Delta_{+}$and $\alpha+\beta \in \Delta$, then $\alpha+\beta \in \Delta_{+}$.

(2) If $\alpha \in \Delta$, then either $\alpha \in \Delta_{+}$or $-\alpha \in \Delta_{+}$.

(3) If $\alpha \in \Delta_{+}$, then $-\alpha \notin \Delta_{+}$.

(See 1.2 for some examples of sets of positive roots.) A subalgebra $\mathfrak{b}$ of $\hat{\mathfrak{g}}$ is a Borel subalgebra if

$$
\mathfrak{b}=\mathfrak{H} \oplus\left(\oplus_{\alpha \in \Delta_{+}} \mathfrak{g}_{\alpha}\right)
$$

for some set of positive of roots $\Delta_{+}$.

We now introduce some subalgebras of $\hat{\mathfrak{g}}$. Let $X \subset \Pi, \dot{\Delta}^{X}$ the subroot system generated by $X$ and $\dot{\Delta}_{ \pm}^{X}=\dot{\Delta}^{X} \cap \dot{\Delta}_{ \pm} . X$ determines a reductive subalgebra $\dot{\mathfrak{m}}$ of $\dot{\mathfrak{g}}$ :

$$
\dot{\mathfrak{m}}=\dot{\mathfrak{m}}^{X}=\dot{\mathfrak{m}}_{-} \oplus \mathfrak{h} \oplus \dot{\mathfrak{m}}_{+}
$$

where $\dot{\mathfrak{m}}_{ \pm}=\bigoplus_{\alpha \in \dot{\Delta}_{ \pm}^{x}} \dot{\mathfrak{g}}_{\alpha} . X$ also determines a nilradical

$$
\dot{\mathfrak{u}}_{ \pm}=\dot{\mathfrak{u}}_{ \pm}^{X}=\oplus_{\alpha \in \dot{\Delta}_{ \pm} \backslash \dot{\Delta} x} \dot{\mathfrak{g}}_{\alpha}
$$

Consequently the decomposition of $\dot{\mathfrak{g}}=\dot{\mathfrak{u}}_{-} \oplus \dot{\mathfrak{m}} \oplus \dot{\mathfrak{u}}_{+}$induces a decomposition of $\hat{\mathfrak{g}}$;

$$
\hat{\mathfrak{g}}=\mathfrak{u}_{-} \oplus \hat{\mathfrak{m}} \oplus \mathfrak{u}_{+}
$$

where

$$
\hat{\mathfrak{m}}=\hat{\mathfrak{m}}^{X}=L(\dot{\mathfrak{m}}) \oplus \mathbb{C} c \oplus \mathbb{C} d \quad \text { and } \quad \mathfrak{u}_{ \pm}=\mathfrak{u}_{ \pm}^{X}=L\left(\dot{\mathfrak{u}}_{ \pm}\right) .
$$

We also set

$$
\mathfrak{m}_{ \pm}^{X}=\left(\dot{\mathfrak{m}}^{X} \otimes \mathbb{C}\left[t^{ \pm 1}\right] t^{ \pm 1}\right) \oplus \dot{\mathfrak{m}}_{ \pm}
$$

so that

$$
\hat{\mathfrak{m}}^{X}=\mathfrak{m}_{-}^{X} \oplus \mathfrak{H} \oplus \mathfrak{m}_{+}^{X}
$$

Since $\dot{\mathfrak{m}}$ is reductive we have $\dot{\mathfrak{m}}=\mathfrak{h}^{X} \oplus \dot{\mathfrak{k}}$ where $\dot{\mathfrak{k}}$ is semisimple and $\mathfrak{h}^{X}:=\left\{h \in \mathfrak{h} \mid \alpha(h)=0\right.$ for all $\left.\alpha \in \dot{\Delta}^{X}\right\}$ is the center of $\dot{\mathfrak{m}}$. Moreover

$$
\dot{\mathfrak{k}}=\dot{\mathfrak{k}}^{X}=\dot{\mathfrak{m}}_{-} \oplus \mathfrak{h}_{X} \oplus \dot{\mathfrak{m}}_{+}
$$


where $\mathfrak{h}_{X}=\sum_{\alpha \in \dot{\Delta}_{+}^{X}}\left[\dot{\mathfrak{g}}_{\alpha}, \dot{\mathfrak{g}}_{-\alpha}\right]$. From $\dot{\mathfrak{k}}$ we can also construct the affine Kac-Moody algebra

$$
\hat{\mathfrak{k}}=L(\dot{\mathfrak{k}}) \oplus \mathbb{C} \boldsymbol{c} \oplus \mathbb{C} d \oplus \mathfrak{h}^{X}
$$

and define

$$
\mathfrak{k}_{ \pm}=\left(\dot{\mathfrak{k}} \otimes \mathbb{C}\left[t^{ \pm 1}\right] t^{ \pm 1}\right) \oplus \dot{\mathfrak{m}}_{ \pm} .
$$

The difference between $\hat{\mathfrak{t}}$ and $\hat{\mathfrak{m}}$ is that $\hat{\mathfrak{m}}$ contains $\mathfrak{h}^{X} \otimes \mathbb{C}\left[t^{ \pm 1}\right] t^{ \pm 1}$ whereas $\hat{\mathfrak{k}}$ does not. To sum it up we have defined three algebras, $\hat{\mathfrak{k}} \subset \hat{\mathfrak{m}} \subset \hat{\mathfrak{g}}$ and our goal is to relate certain composition series of Verma type modules for $\hat{\mathfrak{g}}$ to those for $\hat{\mathfrak{k}}$. In order to introduce these modules we now define the chosen Borel subalgebra for $\hat{\mathfrak{g}}$. Set

$$
\mathfrak{n}_{ \pm}=\mathfrak{n}_{ \pm}^{X}=\mathfrak{m}_{ \pm}^{X} \oplus \mathfrak{u}_{ \pm}^{X}, \quad \mathfrak{b}_{ \pm}=\mathfrak{b}_{ \pm}^{X}=\mathfrak{H} \oplus \mathfrak{n}_{ \pm}^{X}, \quad \mathfrak{p}_{ \pm}=\mathfrak{p}_{ \pm}^{X}=\hat{\mathfrak{m}}^{X} \oplus \mathfrak{u}_{ \pm}^{X} .
$$

One can check that $\mathfrak{b}_{ \pm}$is a Borel subalgebra of $\hat{\mathfrak{g}}$ (see 1.2 below for the appropriate set of positive roots).

Observe that since $\left[\dot{\mathfrak{m}}^{X}, \dot{\mathfrak{u}}_{ \pm}^{X}\right] \subset \dot{\mathfrak{u}}_{ \pm}^{X}$, we have

$$
\left[\mathfrak{b}_{ \pm}^{X}, \mathfrak{u}_{ \pm}^{X}\right] \subset \mathfrak{u}_{ \pm}^{X} \quad \text { and } \quad\left[\hat{\mathfrak{m}}^{X}, \mathfrak{u}_{ \pm}^{X}\right] \subset \mathfrak{u}_{ \pm}^{X}
$$

Note also

- If $X=\emptyset$, then $\dot{\mathfrak{m}}^{X}=\mathfrak{h}, \dot{\mathfrak{u}}_{ \pm}^{X}=\dot{\mathfrak{n}}_{ \pm}$, and $\mathfrak{b}_{+}=\mathfrak{b}^{\text {nat }}$ is the natural Borel subalgebra of $\hat{\mathfrak{g}}$ (see [7]).

- If $X=\Pi$, then $\dot{\mathfrak{m}}^{X}=\dot{\mathfrak{g}}, \dot{\mathfrak{u}}_{ \pm}^{X}=0$ and $\mathfrak{b}_{+}^{X}=\mathfrak{b}_{+}^{\text {st }}$ is the standard Borel subalgebra.

1.2. The representation theory of Verma modules induced from the standard Borel subalgebra has been well examined by other authors (see [8], and [9] and their references). In this article we will relate the representation theory of Verma modules constructed from $\mathfrak{b}_{+}^{X}$ for $X \neq \Pi$ (in particular $\mathfrak{h}^{X} \neq 0$ and $\dot{\mathfrak{u}}_{ \pm}^{X} \neq 0$ ) with Verma modules for standard Borel subalgebras. This study will include the representations induced from the natural Borel subalgebra as a special case and consequently can be viewed as a generalization of Furtony's work [4] where the case $\hat{\mathfrak{g}}=\widehat{s l_{2}(\mathbb{C})}$ is analyzed.

Define

$$
\begin{aligned}
& \Delta_{+}^{X}=\left\{\alpha+n \delta \mid \alpha \in \dot{\Delta}_{+} \backslash \dot{\Delta}_{+}^{X}, n \in \mathbb{Z}\right\} \\
& \cup\left\{\alpha+n \delta \mid \alpha \in \dot{\Delta}^{X} \cup\{0\}, n \in \mathbb{Z}_{>0}\right\} \cup \dot{\Delta}_{+}^{X}
\end{aligned}
$$




$$
\Delta_{ \pm}\left(\hat{\mathfrak{m}}^{X}\right)=\dot{\Delta}_{ \pm}^{X} \cup\left\{\alpha+n \delta \mid \alpha \in \dot{\Delta}^{X} \cup\{0\}, \pm n \in \mathbb{Z}_{>0}\right\}
$$

and

$$
\Delta\left(\mathfrak{u}_{ \pm}\right)=\Delta_{ \pm}\left(\mathfrak{u}^{X}\right)=\left\{\alpha+n \delta \mid \alpha \in \dot{\Delta}_{ \pm} \backslash \dot{\Delta}^{X}, n \in \mathbb{Z}\right\} .
$$

Every positive set of roots of $\Delta$ is $W \times\{ \pm 1\}$ conjugate to one of the sets $\Delta_{+}^{X}$ where $W$ is the Weyl group for $\hat{\mathfrak{g}}$ (see [7], the action of $\{ \pm 1\}$ is on $\delta$ ). Let $Q_{+}^{X}$ (resp. $Q_{+}(\mathfrak{m})$ ) denote the monoid in $\mathfrak{H}^{*}$ generated by $\Delta_{+}^{X}\left(\operatorname{resp} . \Delta_{+}\left(\hat{\mathfrak{m}}^{X}\right)\right)$. Define $\lambda<^{X} \mu$ if $\mu-\lambda \in Q_{+}^{X}$. Let $\dot{Q}$ (respectively $\dot{Q}_{+}, \dot{Q}_{+}(\dot{\mathfrak{m}})$ ) denote the root lattice of $\dot{\Delta}$ (monoid generated by $\dot{\Delta}_{+}, \dot{\Delta}_{+}^{X}$ respectively).

1.3. For $V$ a $\hat{\mathfrak{g}}$-module and $\lambda \in \mathfrak{H}^{*}$, let $V_{\lambda}:=\{v \in V \mid h v=$ $\lambda(h) v$ for all $h \in \mathfrak{H}\}$ be the $\lambda$-th weight space of $V$. Let $P(V)=$ $\left\{\lambda \in \mathfrak{H}^{*} \mid V_{\lambda} \neq 0\right\}$ be the set of weights of $V$ and define $D_{X}(\lambda)=$ $\left\{\mu \in \mathfrak{H}^{*} \mid \mu \leq^{X} \lambda\right\}$. The category $\mathcal{O}^{X}=\mathcal{O}^{X}(\hat{\mathfrak{g}})$ is defined as follows. Objects of $\mathcal{O}^{X}$ are $\mathfrak{H}$-diagonalizable $\hat{\mathfrak{g}}$-modules such that there exists a finite number of elements $\lambda_{1}, \ldots, \lambda_{s} \in \mathfrak{H}^{*}$ with

$$
P(V) \subset \bigcup_{i=1}^{s} D_{X}\left(\lambda_{i}\right)
$$

and $\operatorname{dim} V_{\lambda}$ is at most countable. If $A$ and $B$ are objects in $\mathcal{O}^{X}$ then the set of morphisms from $A$ to $B$ is the set of all $\hat{\mathfrak{g}}$-module homomorphisms. Note that all subquotients, direct sums and tensor products of a finite number of modules in $\mathcal{O}^{X}$ are in $\mathcal{O}^{X}$. We define the categories $\mathcal{O}^{X}(\hat{\mathfrak{m}})$ and $\mathcal{O}^{X}(\hat{\mathfrak{k}})$ in an analogous manner except we replace $Q_{+}^{X}$ in the definition of $<^{X}$ by $Q_{+}^{X}(\hat{\mathfrak{m}})$ and we require that $\operatorname{dim} V_{\lambda}<\infty$ for all $\lambda \in \mathfrak{H}^{*}$ and all modules $V$ in $\mathcal{O}^{X}(\hat{\mathfrak{m}})$ and $\mathcal{O}^{X}(\hat{\mathfrak{k}})$. For $M \in \mathcal{O}^{X}$ and $n \in \mathbb{C}$ define $M^{(n)}=\{m \in M \mid d . m=n m\} ;$ then since $M$ is $\mathfrak{H}$-diagonalizable, we have $M=\oplus_{n \in \mathbb{C}} M^{(n)}$.

1.4. We will now construct some objects in $\mathcal{O}^{X}$. For $L$ a Lie algebra over $\mathbb{C}$, let $U(L)$ denote the universal enveloping algebra of $L$. For $\lambda \in \mathfrak{H}^{*}$ define

$$
M^{X}(\lambda)=U(\hat{\mathfrak{g}}) \otimes_{U\left(\mathfrak{b}_{+}^{X}\right)} \mathbb{C}_{\lambda}
$$

where $\mathbb{C}_{\lambda}$ is the obvious one dimensional $\mathfrak{b}_{+}^{X}$-module. This is the (nonstandard) Verma module for the nonstandard Borel $\mathfrak{b}_{+}^{X}$. We can view $\mathbb{C}_{\lambda}$ as an $\mathfrak{m}_{+}^{X} \oplus \mathfrak{H}$-module by restriction and then define

$$
M_{\mathfrak{m}}^{X}(\lambda)=U\left(\hat{\mathfrak{m}}^{X}\right) \otimes_{U\left(\mathfrak{m}_{+}^{X} \oplus \mathfrak{H}\right)} \mathbb{C}_{\lambda}
$$


and

$$
M_{\mathfrak{k}}^{X}(\lambda)=U\left(\hat{\mathfrak{k}}^{X}\right) \otimes_{U\left(\mathfrak{k}_{+}^{X} \oplus \mathfrak{H}\right)} \mathbb{C}_{\lambda} .
$$

From the standard theory of Verma modules one knows that $M_{\mathfrak{m}}^{X}(\lambda)$ and $M_{\mathfrak{k}}^{X}(\lambda)$ have unique irreducible quotients which we denote by $L_{\mathfrak{m}}^{X}(\lambda)$ and $L_{\mathfrak{k}}^{X}(\lambda)$ respectively. Finally if $N$ is an $\hat{\mathfrak{m}}^{X}$-module then we can make it into a $\mathfrak{p}_{+}^{X}$-module by letting $\mathfrak{u}_{+}^{X}$ act by zero. Inducing up to $\hat{\mathfrak{g}}$ we obtain

$$
U(N):=U(\hat{\mathfrak{g}}) \otimes_{U\left(\mathfrak{p}_{+}^{X}\right)} N .
$$

In the case that $N$ is irreducible we will call $U(N)$ a generalized Verma module for $\hat{\mathfrak{g}}$.

From now on we fix $X \subset \Pi$ and we will often drop $X$ from the notation above except where it might cause some confusion.

Let $P$ be a module for a Lie algebra $\mathfrak{a}$. The intersection of all maximal submodules of $P$ is the radical of $P$ and is denoted by $\operatorname{rad}_{\mathfrak{a}} P$. The proof of the following result is straightforward and is left to the reader.

Proposition 1.5. For $\lambda \in \mathfrak{H}^{*}$, one has for any $\mathfrak{m}$-module subquotient $F$ of $M_{\mathfrak{m}}(\lambda)$

(1) $U(F)=\oplus_{\mu \leq \lambda} U(F)_{\mu}$,

(2) there exists an epimorphism $M(\lambda) \rightarrow N(\lambda)$ where $N(\lambda)=$ $U(F(\lambda))$ and $F(\lambda)$ is the irreducible quotient of $M_{\mathfrak{m}}(\lambda)$,

$$
\operatorname{dim} U(F)_{\mu}= \begin{cases}0 & \text { if } \quad \mu \notin \lambda-Q_{+}, \\ \operatorname{dim} F_{\mu} & \text { if } \quad \mu \in \lambda-Q_{+}(\mathfrak{m}),\end{cases}
$$

(4) $\operatorname{rad} N(\lambda)$ is the unique maximal submodule of $N(\lambda)$.

Using (4) above one sees that $M(\lambda)$ has a unique irreducible quotient which we denote by $L(\lambda)$.

2. Lie algebras with triangular decomposition, contragredient Lie algebras and their representation theory. In this section we consider the structure of $\hat{\mathfrak{m}}, \hat{\mathfrak{k}}$ and their representation theory. More precisely we describe composition series and embeddings of Verma modules for $\hat{\mathfrak{m}}$ in terms of composition series and 
embeddings of Verma modules for $\hat{\mathfrak{k}}$. The relevant references for this section are [5] and [9].

A triangular decomposition of a Lie algebra $\mathfrak{g}$ consists of the following data: three subalgebras $\mathfrak{h}, \mathfrak{g}_{-}$and $\mathfrak{g}_{+}$, an involutive antiautomorphism $\sigma$ of $\mathfrak{g}$ and a free additive semigroup $Q_{+} \subset \mathfrak{h}^{*}$ satisfying

(1) $\mathfrak{g}=\mathfrak{g}_{-} \oplus \mathfrak{h} \oplus \mathfrak{g}_{+}$,

(2) $\mathfrak{g}_{+} \neq 0$ and $\mathfrak{g}_{+}=\oplus_{\alpha \in Q_{+}} \mathfrak{g}_{+\alpha}$ where $\mathfrak{g}_{+\alpha}=\left\{x \in \mathfrak{g}_{+} \mid[h, x]=\right.$ $\alpha(h) x$ for all $h \in \mathfrak{h}\}$,

(3) $\sigma\left(\mathfrak{g}_{+}\right)=\mathfrak{g}_{-}$and $\left.\sigma\right|_{\mathfrak{h}}=1_{\mathfrak{h}}$ and

(4) there exists a basis $\left\{\alpha_{j}\right\}_{j \in J}$ of $Q_{+}$consisting of linearly independent elements of $\mathfrak{h}^{*}$.

A Lie algebra $\mathfrak{g}$ with triangular decomposition is called regular provided $\operatorname{dim} \mathfrak{g}_{\alpha}<\infty$ for all $\alpha \in Q_{+}$. If $\sigma: \hat{\mathfrak{g}} \rightarrow \hat{\mathfrak{g}}$ is the Chevalley involution of $\hat{\mathfrak{g}}$ and if $\dot{\mathfrak{k}}$ is simple then it is straight forward to check that $\left(\mathfrak{h}, \mathfrak{m}_{+}, Q_{+}(\mathfrak{m}),\left.\sigma\right|_{\hat{\mathfrak{m}}}\right)$ and $\left(\mathfrak{h}, \mathfrak{k}_{+}, Q_{+}(\mathfrak{m}),\left.\sigma\right|_{\hat{\mathfrak{k}}}\right)$ are Lie algebras with regular triangular decomposition.

A Lie algebra $\mathfrak{g}$ with triangular decomposition $\left(\mathfrak{h}, \mathfrak{g}_{+}, Q_{+}, \sigma\right)$ is contragredient provided the following is satisfied:

(5) $\operatorname{dim} \mathfrak{g}_{\alpha_{\imath}}=1$ for all $j \in J$,

(6) the elements of $\mathfrak{g}_{\alpha_{j}}, \mathfrak{g}_{-\alpha}, j \in J$ and $\mathfrak{h}$ generate $\mathfrak{g}$,

(7) for all $j \in J, \mathfrak{g}_{\alpha_{\jmath}} \oplus\left[\mathfrak{g}_{\alpha_{j}} \mathfrak{g}_{-\alpha_{\jmath}}\right] \oplus \mathfrak{g}_{-\alpha_{\jmath}}$ is isomorphic to $s l_{2}(\mathbb{C})$ as an algebra and

(8) the sum $\sum_{j \in J}\left[\mathfrak{g}_{\alpha} \mathfrak{g}_{-\alpha_{\jmath}}\right]$ is a direct sum.

For example if $\dot{\mathfrak{k}}$ is a simple Lie algebra then $\hat{\mathfrak{k}}$ is a contragredient Lie algebra with respect to $\left(\mathfrak{h}, \hat{\mathfrak{k}}_{+}, Q_{+}(\mathfrak{m}),\left.\sigma\right|_{\hat{\mathfrak{k}}}\right)$, while $\hat{\mathfrak{m}}$, with respect to $\left(\mathfrak{h}, \hat{\mathfrak{m}}_{+}, Q_{+}(\mathfrak{m}),\left.\sigma\right|_{\hat{\mathfrak{m}}}\right)$, is not if $X \subsetneq \Pi((6)$ above is not satisfied).

The category $\mathcal{O}(\mathfrak{g}, T)$ for a Lie algebra $\mathfrak{g}$ with triangular decomposition $T=\left(\mathfrak{h}, \mathfrak{g}_{+}, Q_{+}, \sigma\right)$ is the category of all $\mathfrak{g}$-modules $M$ such that $M$ is $\mathfrak{h}$-semisimple, the weights of $M$ all lie in a finite union of fans $D(\lambda)=\left\{\mu \in \mathfrak{h}^{*} \mid \mu \in \lambda-Q_{+}\right\}$, and $\operatorname{dim} M_{\lambda}$ is finite for all $\lambda \in \mathfrak{h}^{*}$. As usual the set of morphisms between two objects in this category is the set of all $\mathfrak{g}$-module maps between these objects. Note that in if $\dot{\mathfrak{k}}$ is simple and $T=\left(\mathfrak{h}, \mathfrak{k}_{+}, Q_{+}(\mathfrak{m}),\left.\sigma\right|_{\hat{\mathfrak{k}}}\right)$ or $T=\left(\mathfrak{h}, \mathfrak{m}_{+}, Q_{+}(\mathfrak{m}),\left.\sigma\right|_{\hat{\mathfrak{m}}}\right)$ we have $\mathcal{O}(\mathfrak{g}, T)=\mathcal{O}^{X}(\hat{\mathfrak{k}})$ and $\mathcal{O}(\mathfrak{g}, T)=$ $\mathcal{O}^{X}(\hat{\mathfrak{m}})$ respectively. 
Following [5] we say a Lie algebra $\mathfrak{l}$ is a Heisenberg Lie algebra if

$$
\text { Cent } \mathfrak{l}=[\mathfrak{l}, \mathfrak{l}]=\mathbb{C} z \quad \text { for some } \quad z \in \mathfrak{l} \text {. }
$$

For the rest of this section we will assume $k \in \mathbb{C}$ and $\mathfrak{l}$ is a $\mathbb{Z}$ graded Heisenberg Lie algebra with $\operatorname{dim} \mathfrak{l}_{n}<\infty$ for all $n \in \mathbb{Z}$. Set $\mathfrak{l}_{ \pm}=\oplus_{n>0} \mathfrak{l}_{ \pm n}$ and $\mathfrak{b}_{\mathfrak{l}}=\mathfrak{l}_{+} \oplus \mathbb{C} z$. For $k \in \mathbb{C}$ let $\mathbb{C}_{k}=\mathbb{C} w$ denote the one dimensional $\mathfrak{b}_{\mathfrak{l}}$-module with $\mathfrak{l}_{+} w=0$ and $z w=k w$. One says that a $\mathbb{Z}$-graded $\mathfrak{l}$-module $V$ satisfies property $\mathfrak{C}_{k}$ if (i) $z . v=k v$ for all $v \in V$ and (ii) there exists $N \in \mathbb{Z}$ such that $V_{n}=0$ for $n>N$. Let $\operatorname{Vac}_{\mathfrak{l}_{+}} V=\left\{v \in V \mid \mathfrak{l}_{+} \cdot v=0\right\}$ denote the vacuum space of $V$.

If $X \neq \Pi$ then we define

$$
L=L^{X}:=\left(\mathbb{C}[t] t \otimes \mathfrak{h}^{X}\right) \oplus \mathbb{C} c \oplus\left(\mathbb{C}\left[t^{-1}\right] t^{-1} \otimes \mathfrak{h}^{X}\right) .
$$

$L$ is a Heisenberg algebra with a $\mathbb{Z}$-grading determined by $t$. As for $\mathfrak{l}$ in the previous paragraph, $L$ determines two subalgebras $L_{+}$and $L_{-}$.

We will later need the following result on $\mathbb{Z}$-graded $\mathfrak{l}$-modules $V$.

Theorem 2.1. ([5], Theorem 1.7.3) Let $k \in \mathbb{C} \backslash\{0\}$. Every $\mathfrak{l}-$ module satisfying condition $\mathfrak{C}_{k}$ is completely reducible and in particular is a direct sum of copies of $M(k):=U(\mathfrak{l}) \otimes_{U\left(\mathfrak{b}_{\mathfrak{I}}\right)} \mathbb{C}_{k}$. More precisely if $V$ satisfies condition $\mathfrak{C}_{k}$, then the linear map

$$
f: U(\mathfrak{l}) \otimes_{U\left(\mathfrak{b}_{\mathfrak{l}}\right)} \operatorname{Vac}_{\mathfrak{l}_{+}} V \rightarrow V
$$

given by $u \otimes v \rightarrow u \cdot v$ is an $\mathfrak{l}$-module isomorphism.

REMARK. From the theorem we see that if $V$ satisfies $\mathfrak{C}_{k}$ with $k \neq 0$ then $V$ is free as a $U\left(l_{-}\right)$-module.

We will also need the following useful result.

Proposition 2.2. $\left([\mathbf{3}],[\mathbf{9}]\right.$, Chapter 2). Let $M \in \mathcal{O}^{X}(\mathfrak{m})$ and $\xi \in$ $\mathfrak{h}^{*}$. Then there exists a sequence $0=M_{0} \subset M_{1} \subset \cdots \subset M_{k}=M$ of modules in $\mathcal{O}^{X}(\mathfrak{m})$ and a subset $I \subset\{1, \ldots, k\}$ such that

(1) if $i \in I$ then $M_{i} / M_{i-1} \cong L_{\hat{\mathfrak{m}}}\left(\xi_{i}\right)$ for some $\xi_{i} \geq \xi$ (partially ordered with respect to $\left.Q_{+}\right)$,

(2) if $i \notin I$ then $\left(M_{i} / M_{i-1}\right)_{\mu}=0$ for all $\mu \geq \xi$. 
Such a sequence of submodules of $M$ is called a local composition series of $M$ at $\xi$. The irreducible factors $M_{i} / M_{i-1} \cong L_{\hat{\mathfrak{a}}}\left(\xi_{i}\right)$ (where $\hat{\mathfrak{a}}=\hat{\mathfrak{m}}$ or $\hat{\mathfrak{a}}=\hat{\mathfrak{k}}$ ) occurring in (i) with $\xi_{i} \geq \xi$ are called proper, all others are extraneous. Let $\mu \in \mathfrak{h}^{*}$. The multiplicity of $L_{\hat{\mathfrak{a}}}(\mu)$ in $M$ is the number of proper factors of type $L_{\hat{\mathfrak{a}}}(\mu)$ in any local composition series of $M$ at $\mu$ and is denoted by $\left[M: L_{\hat{\mathfrak{a}}}(\mu)\right]$. (This notion is well defined: [9], Chapter 2.)

Proposition 2.3. Let $\lambda, \mu \in \mathfrak{h}^{*}$. If $\lambda(c) \neq 0$ then

$$
\left[M_{\mathfrak{m}}(\lambda): L_{\mathfrak{m}}(\mu)\right]=\left[M_{\mathfrak{k}}(\lambda): L_{\mathfrak{k}}(\mu)\right]
$$

and the map given by $\phi \mapsto \bar{\phi}:=\left.\phi\right|_{M_{\mathfrak{p}}(\lambda)}$ defines an isomorphism of vector spaces

$$
\text { - }: \operatorname{Hom}_{\hat{\mathfrak{m}}}\left(M_{\mathfrak{m}}(\lambda), M_{\mathfrak{m}}(\mu)\right) \stackrel{\sim}{\rightarrow} \operatorname{Hom}_{\hat{\mathfrak{k}}}\left(M_{\mathfrak{k}}(\lambda), M_{\mathfrak{k}}(\mu)\right) .
$$

Proof. For any $\lambda \in \mathfrak{H}^{*}$ we will view $M_{\mathfrak{k}}(\lambda) \subset M_{\mathfrak{m}}(\lambda)$ in the obvious manner. First suppose $\lambda(c) \neq 0$ and that $0=M_{0} \subset M_{1} \cdots \subset$ $M_{k}=M_{\mathfrak{k}}(\lambda)$ is a local composition series at $\eta$ for the $\hat{\mathfrak{k}}$-module $M_{\mathfrak{k}}(\lambda)$. Define $\bar{M}_{i}=U(\hat{\mathfrak{m}}) M_{i} \subset M_{\mathfrak{m}}(\lambda)$. Since $\left[L_{+}, \hat{\mathfrak{k}}_{-}\right]=0$ one has $\bar{M}_{i}=U\left(L_{-}\right) U(\hat{\mathfrak{k}}) U\left(L_{+}\right) M_{i}=U\left(L_{-}\right) M_{i}$ and thus if we let $L_{+}$act trivially on $M_{i} / M_{i-1}$ then the universal mapping property of the tensor product implies

$$
\bar{M}_{i} / \bar{M}_{i-1} \cong U(\hat{\mathfrak{m}}) \otimes_{U\left(\hat{\mathfrak{k}} \oplus L_{+}\right)}\left(M_{i} / M_{i-1}\right)
$$

as $\hat{\mathfrak{m}}$-modules. If $\left(M_{i} / M_{i-1}\right)_{\mu}=0$ for $\mu \geq \eta$ then $\left(\bar{M}_{i} / \bar{M}_{i-1}\right)_{\mu}=$ 0 since $P\left(\bar{M}_{i} / \bar{M}_{i-1}\right) \subset P\left(M_{i} / M_{i-1}\right)-\mathbb{Z}_{\geq 0} \delta$. We claim that if $M_{i} / M_{i-1} \cong L_{\mathfrak{k}}\left(\mu_{i}\right)$, then $\bar{M}_{i} / \bar{M}_{i-1} \cong L_{\mathfrak{m}}\left(\mu_{i}\right)$. Certainly $L_{\mathfrak{m}}\left(\mu_{i}\right)$ is a quotient of $\bar{M}_{i} / \bar{M}_{i-1}$. Let $0 \neq u \in U(\hat{\mathfrak{m}}) \otimes_{U\left(\hat{\mathfrak{k}} \oplus L_{+}\right)} L_{\mathfrak{k}}\left(\mu_{i}\right)$ be a weight vector. Then we can write

$$
u=\sum_{l, j} \alpha_{l j} u_{l} \otimes v_{j}
$$

where $\left\{u_{l}\right\}_{l \in I}$ and $\left\{v_{j}\right\}_{j \in J}$ are basis elements of weight vectors for $U\left(L_{-}\right)$and $L_{\mathfrak{k}}\left(\mu_{i}\right)$ respectively and $\alpha_{l j} \in \mathbb{C}$. Among all indices $(l, j)$ with $\alpha_{l j} \neq 0$ let $(a, b)$ be such that $v_{b}$ has minimal weight 
(there may be many such pairs of indices). Let $v_{\mu_{i}}$ be a nonzero highest weight vector in $L_{\mathfrak{k}}\left(\mu_{i}\right)$ and suppose $v_{b}$ has weight $\mu_{i}-\xi$ and $0 \neq \xi \in Q_{+}(\mathfrak{m})$. Since $\alpha_{a b} \neq 0$ and the $v_{j}$ are linearly independent one has $\sum_{j \in J^{\prime}} \alpha_{a j} v_{j} \neq 0$ where $J^{\prime}$ is the set of indices $j$ with $v_{j} \in L_{\mathfrak{k}}\left(\mu_{i}\right)_{\mu_{i}-\xi}$. Now $L_{\mathfrak{k}}\left(\mu_{i}\right)$ is irreducible, so there exists $w \in U(\hat{\mathfrak{k}})_{\xi}$ with $w\left(\sum_{j \in J^{\prime}} \alpha_{a j} v_{j}\right)=v_{\mu_{i}}$. The fact that $v_{b}$ has minimal weight implies that $w v_{j}=0$ for $j \notin J^{\prime}$ with $\alpha_{l j} \neq 0$. Thus $w\left(\sum_{j \notin J^{\prime}} \alpha_{a j} v_{j}\right)=0$ and $\left[\hat{\mathfrak{k}}, L_{-}\right]=0$ implies that $w u=\sum_{l}\left[u_{l} \otimes\right.$ $\left.\left(\sum_{j} \alpha_{l j} w v_{j}\right)\right]=\left(\sum_{l} \beta_{l} u_{l}\right) \otimes v_{\mu_{i}}$ where $\beta_{a}=1$ and $\beta_{l} \in \mathbb{C}$. Since $U\left(L_{-}\right) \otimes \mathbb{C} v_{\mu_{i}}$ is irreducible as an $L$-module (Theorem 2.1) one must have that $U(\hat{\mathfrak{m}}) u=U(\hat{\mathfrak{m}}) \otimes_{U\left(\hat{\mathbf{k}} \oplus L_{+}\right)} L_{\mathfrak{k}}\left(\mu_{i}\right)$. This proves the claim. Observe that we have also proven that if $F$ is an irreducible subquotient of $M_{\mathfrak{m}}(\lambda)$ then $F \cong U(\hat{\mathfrak{m}}) \otimes_{U\left(\hat{\mathfrak{k}} \oplus L_{+}\right)} L_{\mathfrak{k}}(\mu)$ for some $\mu \in \mathfrak{H}^{*}$.

Let now $0 \neq u \in \operatorname{Vac}_{\mathfrak{k}_{+}} M_{\mathfrak{k}}(\mu)$, then since $\left[L_{+}, \hat{\mathfrak{k}}_{-}\right]=0$ we have that $u \in \operatorname{Vac}_{\mathfrak{m}_{+}} M_{\mathfrak{m}}(\mu)$. If $v \in \operatorname{Vac}_{\mathfrak{m}_{+}} M_{\mathfrak{m}}(\mu)$ one has

$$
v=\sum_{l, j} \alpha_{l j} u_{l} \otimes v_{j}
$$

where $\left\{u_{l}\right\}$ is as in the preceding paragraph and $\left\{v_{j}\right\}$ is a basis of $M_{\mathfrak{k}}(\mu)$. Since $\left[L_{+}, \hat{\mathfrak{k}}_{-}\right]=0$ and $v \in \operatorname{Vac}_{\mathfrak{m}_{+}} M_{\mathfrak{m}}(\mu)$ we have that $\left[L_{+}, \sum_{l} \alpha_{l j} u_{l}\right]=0$ for all $j$. Using Theorem 2.1 we have $\sum_{l} \alpha_{l j} u_{l} \in \mathbb{C}$ for all $j$ i.e. $v=\sum_{j} \beta_{j} v_{j}$ for some $\beta_{j} \in \mathbb{C}$. Thus $\operatorname{Vac}_{\mathfrak{k}_{+}} M_{\mathfrak{k}}(\mu)=\operatorname{Vac}_{\mathfrak{m}_{+}} M_{\mathfrak{m}}(\mu)$. Using this fact, together with the universal mapping property of Verma modules, the reader can deduce that the map $\left.\phi \mapsto \phi\right|_{M_{\mathfrak{f}}(\lambda)}$ is a bijection from $\operatorname{Hom}_{\hat{\mathfrak{m}}}\left(M_{\mathfrak{m}}(\lambda), M_{\mathfrak{m}}(\mu)\right)$ to $\operatorname{Hom}_{\hat{\mathfrak{k}}}\left(M_{\mathfrak{k}}(\lambda), M_{\mathfrak{k}}(\mu)\right)$.

\section{A basis for $U(L(\dot{\mathfrak{g}}))$.}

3.1. Let $\rho$ be the rank of $\dot{\mathfrak{g}}$ and let $C=\left\{y_{\alpha}, h_{k} \mid \alpha \in \dot{\Delta}, 1 \leq k \leq \rho\right\}$ be a Chevalley basis of $\dot{\mathfrak{g}}$. Let $\left\{x_{j}\right\}_{j=1}^{\mathrm{dim} \dot{\mathfrak{m}}_{+}} \subset C$ be a basis of $\dot{\mathfrak{m}}_{+}$and $\left\{x_{j}\right\}_{j=-\operatorname{dim} \dot{m}_{+}} \subset C$ be a basis of $\dot{\mathfrak{m}}_{-}$. Here we assume that (1) if $x_{i} \in \dot{\mathfrak{g}}_{\beta_{i}}$ and $x_{j} \in \dot{\mathfrak{g}}_{\beta}$, with $\beta_{i}<\beta_{j}$, then $i<j$ and 
(2) if $x_{i} \in \dot{\mathfrak{g}}_{\beta_{\mathfrak{\imath}}}$, then $x_{-i} \in \dot{\mathfrak{g}}_{-\beta_{\mathfrak{i}}}$.

In addition we define $x_{i}<x_{j}$ if $i<j$. Let $\left\{y_{j}\right\}_{j=1}^{\operatorname{dim} \dot{u}_{+}} \subset C$ (resp $\left\{y_{j}\right\}_{j=-\operatorname{dim} \dot{u}_{+}}^{-1} \subset C$ ) be a basis of root vectors of $\dot{\mathfrak{u}}_{+}^{X}$ (resp. $\dot{\mathfrak{u}}_{-}^{X}$ ) satisfying (1) and (2) above. We also define $y_{i}<y_{j}$ if $i<j$. If $z_{i} \in \dot{\mathfrak{g}}_{\alpha}$ is a Chevalley basis element, let $\left\{z_{i}, \check{\alpha}_{i}, z_{-i}\right\}$ be the $s l_{2}(\mathbb{C})$ triple determined by $z_{i}$. Finally we totally order the Chevalley basis $C$ by

$$
y_{f}<x_{g}<h_{i}<h_{j}<x_{k}<y_{l}
$$

if $-\operatorname{dim} \dot{\mathfrak{u}}_{+} \leq f \leq-1,-\operatorname{dim} \dot{\mathfrak{m}}_{+} \leq g \leq-1, \quad 1 \leq i<j \leq \rho$, $1 \leq k \leq \operatorname{dim} \dot{\mathfrak{m}}_{+}$and $1 \leq l \leq \operatorname{dim} \dot{\mathfrak{u}}_{+}$. Note that this is a total ordering on $C$ and is compatible with the usual partial ordering on $\dot{\mathfrak{g}}$ induced by the positive roots of $\dot{\Delta}$.

Next we will order a basis of $L(\dot{\mathfrak{g}})$ using (1) and (2) above. Set

$$
x(m)=x \otimes t^{m}
$$

for $x \in \dot{\mathfrak{g}}$ and $m \in \mathbb{Z} . \quad L(\dot{\mathfrak{g}})$ has a basis $B(L(\dot{\mathfrak{g}})):=\{x(m) \mid x \in$ $C, m \in \mathbb{Z}\}$ which we totally order by defining

$$
x(m)<y(n)
$$

if either (1) $m<n$ or (2) $m=n$ and $x<y$. (Note that if $x(m) \epsilon$ $L(\dot{\mathfrak{g}})_{\alpha}$ and $y(n) \in L(\dot{\mathfrak{g}})_{\beta}$ with $\alpha<\beta$ and $x$ and $y$ are in $C$, then $x(m)<y(n)$.) In particular $\mathfrak{u}_{-}$has a basis

$$
B\left(\mathfrak{u}_{-}\right)=B(L(\dot{\mathfrak{g}})) \cap \mathfrak{u}_{-}=\left\{y_{i}(m) \mid 1 \leq-i \leq \operatorname{dim} \dot{\mathfrak{u}}_{+}, m \in \mathbb{Z}\right\} .
$$

In fact, if $\mathfrak{a}$ is any subalgebra of $L(\dot{\mathfrak{g}})$, then we define

$$
B(\mathfrak{a})=B(L(\dot{\mathfrak{g}})) \cap \mathfrak{a} .
$$

In the following we will use the multi-index notation $\mathbf{a}=\left(a_{1}, \ldots, a_{r}\right)$ for $a_{i} \in \mathbb{Z}$. If $r \geq 1$ and $(\mathbf{i}, \mathbf{m}, \mathbf{p})=\left(i_{1}, \ldots, i_{r}, m_{1}, \ldots, m_{r}, p_{1}, \ldots, p_{r}\right) \in$ $\mathbb{Z}^{3 r}$, then set $\bar{z}=z_{\mathbf{i}, \mathbf{m}, \mathbf{p}}=z_{i_{1}}\left(m_{1}\right)^{p-i} \cdots z_{i_{r}}\left(m_{r}\right)^{p_{r}}$. We will use the convention

$$
\begin{array}{ll}
\bar{z}=z_{\mathbf{i}, \mathbf{m}, \mathbf{p}}=z_{i_{1}}\left(m_{1}\right)^{p-i} \cdots z_{i_{r}}\left(m_{r}\right)^{p_{r}}=0 & \text { if } p_{i}<0 \text { for some } i \text { and } \\
\bar{z}=z_{\mathbf{i}, \mathbf{m}, \mathbf{p}}=z_{i_{1}}\left(m_{1}\right)^{p-i} \cdots z_{i_{r}}\left(m_{r}\right)^{p_{r}}=1 & \text { if } p_{i}=0 \text { for all } i .
\end{array}
$$

Now define

$$
\bar{z}^{\hat{j}}=z_{i_{1}}\left(m_{1}\right)^{p_{1}} \cdots z_{i_{j}}\left(m_{j}\right)^{p_{j}-1} \cdots z_{i_{r}}\left(m_{r}\right)^{p_{r}}
$$


for $1 \leq j \leq r$. For $1 \leq j \leq \xi \leq r$ define

$$
\bar{z}^{\hat{\xi} \hat{j}}=\bar{z}^{\hat{j} \hat{\xi}}= \begin{cases}z_{i_{1}}\left(m_{1}\right)^{p_{1}} \cdots z_{i_{\jmath}}\left(m_{j}\right)^{p_{j}-1} \cdots z_{i_{\xi}}\left(m_{\xi}\right)^{p_{\xi}-1} & \cdots z_{i_{r}}\left(m_{r}\right)^{p_{r}} \\ z_{i_{1}}\left(m_{1}\right)^{p_{1}} \cdots z_{i_{j}}\left(m_{j}\right)^{p_{j}-2} \cdots z_{i_{r}}\left(m_{r}\right)^{p_{r}} & \text { if } j \neq \xi \\ \text { if } j=\xi\end{cases}
$$

In other words $\bar{z}^{\hat{\xi} \hat{j}}$ is essentially just $\bar{z}$ where we've decreased the exponents $p_{\xi}$ and $p_{j}$ by one.

By the Poincaré-Birkhoff-Witt Theorem $U\left(\mathfrak{u}_{-}\right)$has a basis

$$
\begin{aligned}
B\left(U\left(\mathfrak{u}_{-}\right)\right):=\left\{y_{\mathbf{i}, \mathbf{m}, \mathbf{p}}=\right. & y_{i_{1}}\left(m_{1}\right)^{p-i} \cdots y_{i_{r}}\left(m_{r}\right)^{p_{r}} \mid y_{i_{j}}\left(m_{j}\right) \\
& \left.<y_{i_{j+1}}\left(m_{j+1}\right) y_{i_{j}}\left(m_{j}\right) \in B\left(\mathfrak{u}_{-}\right)\right\} \cup\{1\} .
\end{aligned}
$$

If $f \in U\left(\mathfrak{u}_{-}\right)$is a polynomial in reduced words from $B\left(U\left(\mathfrak{u}_{-}\right)\right)$then we define the degree of $f$ to be the maximum length of any word appearing in $f$ and let $U\left(\mathfrak{u}_{-}\right)_{(p)}$ be the set of polynomials of degree $p$ or less. Thus if $\bar{y}=y_{i_{1}}\left(m_{1}\right)^{p-i} \cdots y_{i_{r}}\left(m_{r}\right)^{p_{r}}$ then $\operatorname{deg} \bar{y}=\sum_{i} p_{i}$. Define also $U\left(\mathfrak{u}_{-}\right)_{(p)}=0$ for $p<0$. We totally order the words in $B\left(U\left(\mathfrak{u}_{-}\right)\right)$by degree and then among words of equal degree we order reverse lexicographically. Moreover if $w=\mu z_{\mathbf{i}, \mathbf{m}, \mathbf{p}}$ and $v=\xi y_{\mathbf{a}, \mathbf{b}, \mathbf{c}}$ with $z_{\mathbf{i}, \mathbf{m}, \mathbf{p}}<y_{\mathbf{a}, \mathbf{b}, \mathbf{c}}$ and $\mu, \xi \in \mathbb{C} \backslash\{0\}$, then we also write $w<v$. For example $y_{-5}(7)<y_{-2}(3)^{2} y_{-1}(4)^{3}<y_{-2}(3) y_{-1}(5)^{4}$.

REMARK 3.2. Suppose $\mathfrak{t}$ is a Lie algebra over $\mathbb{C}$ and $\mathfrak{a} \subset \mathfrak{t}$ is a subalgebra with basis $\left\{y_{l}\right\}_{l \in L}$. Let $\left\{z_{j}\right\}_{j \in J}$ be a basis complementary to $\left\{y_{l}\right\}_{l \in L}$ (i.e. $\left\{y_{l}\right\}_{l \in L} \cup\left\{z_{j}\right\}_{j \in J}$ is a basis of $\mathfrak{t}$ ) with $J$ totally ordered by a relation $<_{J}$. Then it is well known (see [HS]) that $U(\mathfrak{t})$ is a free right $U(\mathfrak{a})$-module with $U(\mathfrak{a})$-basis $\left\{z_{j_{1}}^{m_{1}} \cdots z_{j_{k}}^{m_{k}} \mid m_{j} \in\right.$ $\mathbb{Z}_{>0}$ and $\left.j_{i}<_{J} j_{i+1}\right\} \cup\{1\}$. This in particular implies that if $z_{j_{1}}^{m_{1}} \cdots z_{j_{k}}^{m_{k}} a=z_{l_{1}}^{n_{1}} \cdots z_{l_{r}}^{n_{r}} a^{\prime}$ with $l_{1}<_{J} \cdots<_{J} l_{r}, n_{i} \in \mathbb{Z}_{>0}$, then $m_{i}=n_{i}, j_{i}=l_{i}$ for all $i$ and $a=a^{\prime}$.

In addition if $F$ is an $\hat{\mathfrak{m}}$-module with basis $\left\{x_{j}\right\}_{j \in J}$ then by the Poincaré-Birkhoff-Witt theorem

$$
U(F)=U(\hat{\mathfrak{g}}) \otimes_{U(\hat{\mathfrak{m}})} F \cong U\left(\mathfrak{u}_{-}\right) \otimes F
$$

has a basis consisting of vectors of the form $y_{i_{1}}\left(m_{1}\right)^{p-i} \cdots y_{i_{r}}\left(m_{r}\right)^{p_{r}} x_{j}$. We order this basis lexicographically by declaring $y_{\mathbf{i}, \mathbf{m}, \mathbf{p}} x_{k}<y_{\mathbf{a}, \mathbf{b}, \mathbf{c}} x_{l}$ if either $y_{\mathbf{i}, \mathbf{m}, \mathbf{p}}<y_{\mathbf{a}, \mathbf{b}, \mathbf{c}}$ or if $y_{\mathbf{i}, \mathbf{m}, \mathbf{p}}=y_{\mathbf{a}, \mathbf{b}, \mathbf{c}}$ but $k<l$. In addition 
we will later use the fact that $U(F)$ is a free $U\left(\mathfrak{u}_{-}\right)$-module with $U\left(\mathfrak{u}_{-}\right)$-basis $\left\{x_{j}\right\}_{j \in J}$.

Finally if

$$
u=\sum u_{\mathbf{i}, \mathbf{m}, \mathbf{p}}^{j} y_{\mathbf{i}, \mathbf{m}, \mathbf{p}} x_{j}
$$

then we let

$$
\operatorname{LinSp}(u)=\operatorname{Span}\left\{y_{\mathbf{i}, \mathbf{m}, \mathbf{p}} x_{j} \mid u_{\mathbf{i}, \mathbf{m}, \mathbf{p}}^{j} \neq 0\right\}
$$

be the span over $\mathbb{C}$ of the basis elements $y_{\mathbf{i}, \mathbf{m}, \mathbf{p}} x_{j}$ occuring in the decomposition of $u$ above that have nonzero coefficients.

4. An irreducibility criterion for subquotients of $M(\lambda)$. 4.1. In this section we will assume that $\lambda(c) \neq 0$ and $F$ is an $\hat{\mathfrak{m}}$-subquotient of $M_{\mathfrak{m}}(\lambda)$. The main result in this section is an irreducibility criterion for

$$
U(F):=U(\hat{\mathfrak{g}}) \otimes_{U(\hat{\mathfrak{m}})} F \quad(\text { see } 4.6) .
$$

There are a couple of technical lemmas that will be used throughout the case by case proof of this result and it is best to get them out the way before diving into the analysis.

Nolan Wallach kindly provided the author with a nice proof of the first technical result:

LEMMA 4.2. If $\alpha \in \Delta\left(\dot{\mathfrak{u}}_{-}\right)$is a nonsimple root and $y \in \dot{\mathfrak{g}}_{-\alpha}$ is nonzero, then there exists $z \in B\left(\dot{\mathfrak{n}}_{+}\right)$such that $0 \neq[z, y] \in \dot{\mathfrak{u}}_{-}$.

Proof. Recall that we always assume $X \neq \Pi$ so that $\dot{\Delta}_{+} \backslash \dot{\Delta}^{X} \neq \emptyset$. Set $\rho=\operatorname{rank} \dot{\mathfrak{g}}$. We will prove this result by induction on $\rho$. If $\rho=1$, then $X=\emptyset$ and the result follows.

Suppose now $\rho>1$. Let $\left\{\alpha_{i}\right\}_{i=1}^{\rho}=\Pi$ be Dynkin's enumeration of the set of simple roots in $\dot{\Delta}_{+}($see $[8])$ and let $n_{i} \in \mathbb{Z}$ be nonnegative integers such that $\alpha=\sum_{i=1}^{\rho}-n_{i} \alpha_{i}$. Recall that the support of $\alpha$, denoted by $\operatorname{supp}(\alpha)$, is the set of all indices $i$ such that $n_{i} \neq 0$. If $|\operatorname{supp}(\alpha)|<\rho$ then we have that $\alpha$ lies in a subalgebra of $\dot{\mathfrak{g}}$ that is of smaller rank and by induction the result follows. Consequently we may assume that $|\operatorname{supp}(\alpha)|=\rho$ so that $n_{i}>0$ for all $1 \leq i \leq \rho$. Let 
$(, \quad)$ be the Killing form on $\mathfrak{h}^{*}$ and for any $\beta \in \dot{\Delta}$, let $\check{\beta}=2 \beta /(\beta, \beta)$ denote its coroot.

We first consider the case that $n_{k}>1$ for some $1 \leq k \leq \rho$. Here $\dot{\mathfrak{g}}$ cannot be of type $A_{l}$ as the longest root of $A_{l}$ is of the form $\sum_{i=1}^{\rho} \alpha_{i}$ and thus $n_{i}=1$ for all $i$. Suppose that $\left(\alpha, \check{\alpha}_{i}\right) \geq 0$ for all indices $i$ such that $n_{i}>1$. We will prove by induction on $\rho$ that this is not possible. Consider the equality

$$
2=(\alpha, \check{\alpha})=-\sum_{i: n_{\imath}>1} n_{i}\left(\alpha_{i}, \check{\alpha}\right)-\sum_{i: n_{\imath}=1} n_{i}\left(\alpha_{i}, \check{\alpha}\right) \text {. }
$$

If $\left(\alpha_{i}, \check{\alpha}\right) \geq 0$ for all indices $i$ with $n_{i}=1$, then we obtain a contradiction. Hence there exists an index $j$ with $n_{j}=1$ and $\left(\alpha, \check{\alpha}_{j}\right)<0$. Then

$$
\left(\alpha, \check{\alpha}_{j}\right)=-\sum_{i \neq j} n_{i}\left(\alpha_{i}, \check{\alpha}_{j}\right)-2<0
$$

so that

$$
0<-\sum_{i \neq j} n_{i}\left(\alpha_{i}, \check{\alpha}_{j}\right)<2
$$

(this is nonzero as the node $j$ is connected to at least one other node). Since $n_{i}>0$ for all $i$ this implies $\left(\alpha, \check{\alpha}_{j}\right)=-1$ and if $k$ is such that $n_{k} \geq 2$ then $\left(\alpha_{k}, \check{\alpha}_{j}\right)=0$. Consequently $\alpha+\alpha_{j}$ is a negative root and $\left|\operatorname{supp}\left(\alpha+\alpha_{j}\right)\right|=\left|\operatorname{supp}\left(\alpha_{j}\right)\right|-1$ since $n_{j}=1$. Now induction on $\rho$ implies that there exists a $k$ with $n_{k} \geq 2$ such that $\alpha+\alpha_{j}+\alpha_{k}$ is a negative root.

The calculation

$$
\left(\alpha+\alpha_{j}+\alpha_{k}, \check{\alpha}_{j}\right)=\left(\alpha, \check{\alpha}_{j}\right)+2+\left(\alpha_{k}, \check{\alpha}_{j}\right)=-1+2=1
$$

$\left(\right.$ recall $\left(\alpha_{l}, \check{\alpha}_{j}\right)=0$ for $l$ with $\left.n_{l}>1\right)$. Hence $\alpha+\alpha_{k}=\alpha+\alpha_{j}+\alpha_{k}-\alpha_{j}$ is a negative root.

Now we have reduced to the case $\alpha=-\sum_{i=1}^{\rho} \alpha_{i}$. By considering the Dynkin diagram of $\dot{\mathfrak{g}}$ one can deduce that there are at least two indices $i_{1} \neq i_{2}$ such that $\alpha+\alpha_{i_{1}}$ and $\alpha+\alpha_{i_{2}}$ are roots in $\dot{\Delta}$. If $\alpha_{i_{1}}, \alpha_{i_{2}} \in \Delta\left(\dot{\mathfrak{u}}_{+}\right)$, then we choose $0 \neq z \in\left(\dot{\mathfrak{u}}_{+}\right)_{\alpha_{i_{1}}}$ and the result follows as $\operatorname{supp}\left(\alpha+\alpha_{i_{1}}\right)$ contains an index $j$ with $\alpha_{j} \in \Delta\left(\dot{\mathfrak{u}}_{+}\right)$so that $\alpha+\alpha_{i_{1}} \in \Delta\left(\dot{\mathfrak{u}}_{-}\right)$. If $\alpha_{i_{1}} \in \Delta_{+}(\dot{\mathfrak{m}})$, then since $\operatorname{supp}\left(\alpha+\alpha_{i_{1}}\right)$ contains 
an index $j$ with $\alpha_{j} \in \Delta\left(\dot{\mathfrak{u}}_{+}\right)$, there is a basis element $z \in\left(\dot{\mathfrak{m}}_{+}\right)_{\alpha_{\mathfrak{i}_{1}}}$ with $0 \neq[z, y] \in \dot{\mathfrak{u}}_{-}$.

The second technical result will help consolidate later calculations. Before we state it though we need to introduce some more notation: Suppose

$$
y=y_{i_{1}}\left(m_{1}\right)^{p_{1}} \cdots y_{i_{r}}\left(m_{r}\right)^{p_{r}} \quad, \quad e \in \mathbb{Z} \quad \text { and } \quad z \in \dot{\mathfrak{g}} .
$$

Define

$$
\begin{aligned}
& J^{c}=J^{c}(z, \bar{y})=\left\{1 \leq j \leq r \mid 0 \neq\left[y_{i_{j}}, z\right] \in \dot{\mathfrak{u}}_{+} \oplus \dot{\mathfrak{m}}\right\} \\
& J_{-}=J_{-}(z, \bar{y})=\left\{1 \leq j \leq r \mid 0 \neq\left[y_{i_{\jmath}}, z\right] \in \dot{\mathfrak{u}}_{-}\right\} \\
& J_{-}^{l}=J_{-}^{l}(z, \bar{y}, e)=\left\{j \in J_{-} \mid y_{i_{\jmath}}\left(m_{j}\right) \geq\left[z, y_{i_{\jmath}}\right]\left(e+m_{j}\right)\right\} \\
& J_{-}^{r}=J_{-}^{r}(z, \bar{y}, e)=\left\{j \in J_{-} \mid y_{i_{\jmath}}\left(m_{j}\right)<\left[z, y_{i_{j}}\right]\left(e+m_{j}\right)\right\} .
\end{aligned}
$$

LEMMA 4.3. Suppose $\lambda(c) \neq 0$ and $F$ is a subquotient of $M_{\mathfrak{m}}(\lambda)$. Let $\bar{x}$ be a nonzero weight vector in $F, z \in \dot{\mathfrak{g}}, e \in \mathbb{Z}$, and $\bar{y}=$ $y_{i_{1}}\left(m_{1}\right)^{p_{1}} \cdots y_{i_{r}}\left(m_{r}\right)^{p_{r}}$ with $\operatorname{deg} \bar{y} \geq 1$. If either (1) $z \in \dot{\mathfrak{u}}_{+}$and $e \ll 0$ or (2) $e \gg 0$ and $z \in \dot{\mathfrak{m}}_{+}$, then

$$
\begin{aligned}
z(e) \bar{y} \bar{x} \equiv & \sum_{j \in J_{-}^{l}} p_{j}\left[z, y_{i_{j}}\right]\left(e+m_{j}\right) \bar{y}^{\hat{j}} \bar{x}+\sum_{j \in J_{-}^{r} \cup J^{c}} p_{j} \bar{y}^{\hat{j}}\left[z, y_{i_{j}}\right]\left(e+m_{j}\right) \bar{x} \\
& +\sum_{j \in J_{-}^{l} \backslash\{1\}} \sum_{\xi=1}^{j-1} p_{j} p_{\xi}\left[y_{i_{\xi}},\left[z, y_{i_{j}}\right]\right]\left(e+m_{j}+m_{\xi}\right) \bar{y}^{\hat{j} \hat{\xi}} \bar{x} \\
& +\sum_{j \in\left(J_{-}^{r} \cup J^{c}\right) \backslash\{r\}} \sum_{\xi=j+1}^{r} p_{j} p_{\xi}\left[\left[z, y_{i_{\jmath}}\right], y_{i_{\xi}}\right]\left(e+m_{j}+m_{\xi}\right) \bar{y}^{\hat{j} \hat{\xi}} \bar{x} \\
& +\sum_{1 \leq j \leq r} \epsilon_{j}\left[y_{i_{j}},\left[z, y_{i_{j}}\right]\right]\left(e+2 m_{j}\right) \bar{y}^{\hat{j} \hat{j}} \bar{x} \bmod U\left(\mathfrak{u}_{-}\right)_{(p-2)} \otimes F
\end{aligned}
$$

where

$$
\epsilon_{j}=\left\{\begin{aligned}
-p_{j}\left(p_{j}-1\right) / 2 & \text { if } j \in J^{c} \cup J_{-}^{r} \\
p_{j}\left(p_{j}-1\right) / 2 & \text { if } j \in J_{-}^{l}
\end{aligned}\right.
$$

and $p=\operatorname{deg} \bar{y}=\sum_{i} p_{i}$.

We use the convention $x^{0}=1$ in $U(\hat{\mathfrak{g}})$ for any nonzero element $x \in \hat{\mathfrak{g}}$ and if an index set for some summation is empty, then we take the summation to be zero. 
Proof. First observe that if $e \gg 0$ or $e \ll 0$ then $\left[\left[z(e), y_{i_{j}}\left(m_{j}\right)\right]\right.$, $\left.y_{i_{\xi}}\left(m_{\xi}\right)\right]$ and $\left[z(e), y_{i_{j}}\left(m_{j}\right)\right]$ act on any element in $U(F)$ by $\left[\left[z, y_{i_{j}}\right]\right.$, $\left.y_{i_{\xi}}\right]\left(e+m_{j}+m_{\xi}\right)$ and $\left[z, y_{i_{j}}\right]\left(e+m_{j}\right)$ respectively i.e no central term appears. Moreover if either (1) or (2) above holds then $z(e) \bar{x}=0$. Now the Lemma follows from the calculation below.

$$
\begin{aligned}
& z(e) \bar{y} \bar{x}=\sum_{j=1}^{r} \sum_{\alpha=0}^{p_{j}-1} y_{i_{1}}\left(m_{1}\right)^{p_{1}} \cdots y_{i_{j}}\left(m_{j}\right)^{\alpha} \\
& \cdot\left[z, y_{i_{j}}\right]\left(e+m_{j}\right) y_{i_{j}}\left(m_{j}\right)^{p_{j}-\alpha-1} \cdots y_{i_{r}}\left(m_{r}\right)^{p_{r}} \bar{x} \\
& =\sum_{j \in J_{-}^{l}}\left(\sum_{\alpha=0}^{p_{j}-1}\left[z, y_{i}\right]\left(e+m_{j}\right) \bar{y}^{\hat{j}} \bar{x}\right. \\
& +\sum_{\alpha=0}^{p_{j}-1}\left[y_{i_{1}}\left(m_{1}\right)^{p_{1}} \cdots y_{i_{\jmath}}\left(m_{j}\right)^{\alpha},\left[z, y_{i_{j}}\right]\left(e+m_{j}\right)\right] \\
& \left.\cdot y_{i_{j}}\left(m_{j}\right)^{p_{j}-\alpha-1} \cdots y_{i_{r}}\left(m_{r}\right)^{p_{r}} \bar{x}\right) \\
& +\sum_{j \in J_{-}^{r} \cup J^{c}}\left(\sum_{\alpha=0}^{p_{j}-1} \bar{y}^{\hat{j}}\left[z, y_{i}\right]\left(e+m_{j}\right) \bar{x}\right. \\
& +\sum_{\alpha=0}^{p_{j}-1} y_{i_{1}}\left(m_{1}\right)^{p_{1}} \cdots y_{i_{\jmath}}\left(m_{j}\right)^{\alpha}\left[\left[z, y_{i_{j}}\right]\right. \\
& \left.\left.\cdot\left(e+m_{j}\right), y_{i_{j}}\left(m_{j}\right)^{p_{j}-\alpha-1} \cdots y_{i_{r}}\left(m_{r}\right)^{p_{r}}\right] \bar{x}\right) \\
& \equiv \sum_{j \in J_{-}^{l}} p_{j}\left[z, y_{i}\right]\left(e+m_{j}\right) \bar{y}^{\hat{j}} \bar{x} \\
& +\sum_{j \in J_{-}^{l}} \frac{p_{j}\left(p_{j}-1\right)}{2}\left[y_{i_{j}},\left[z, y_{i_{j}}\right]\right]\left(e+2 m_{j}\right) \bar{y}^{\hat{j} \hat{j}} \bar{x} \\
& +\sum_{j \in J_{-} \backslash\{1\}} \sum_{\xi=1}^{j-1} p_{j} p_{\xi}\left[y_{i_{\xi}},\left[z, y_{i_{j}}\right]\right]\left(e+m_{j}+m_{\xi}\right) \bar{y}^{\hat{j} \hat{\xi}_{\bar{x}}} \\
& +\sum_{j \in J_{-}^{r} \cup J^{c}} p_{j} \bar{y}^{\hat{j}}\left[z, y_{i_{j}}\right]\left(e+m_{j}\right) \bar{x}
\end{aligned}
$$




$$
\begin{aligned}
&+ \sum_{j \in J_{-}^{r} \cup J^{c}} \frac{p_{j}\left(p_{j}-1\right)}{2} \bar{y}^{\hat{j} \hat{j}}\left[\left[z, y_{i_{j}}\right], y_{i_{j}}\right]\left(e+2 m_{j}\right) \bar{x} \\
&+\sum_{j \in J_{-}^{r} \cup J^{c} \backslash\{r\}} \sum_{\xi=j+1}^{r} p_{j} p_{\xi}\left[\left[z, y_{i_{j}}\right], y_{i_{\xi}}\right] \\
& \cdot\left(e+m_{j}+m_{\xi}\right) \bar{y}^{\hat{j} \hat{\xi}} \bar{x} \bmod U\left(\mathfrak{u}_{-}\right)_{(p-2)} \otimes F .
\end{aligned}
$$

4.4. If $F$ is an irreducible subquotient of $M_{\mathfrak{m}}(\lambda), F$ is of the form $U(\hat{\mathfrak{m}}) \otimes_{U\left(\hat{\mathfrak{k}} \otimes L_{+}\right)} F^{\prime}$ where $F^{\prime}$ is a subquotient of $M_{\mathfrak{k}}(\lambda)$ (see the observation in the proof of Proposition 2.3). In particular $F$ is a free $U\left(L_{-}\right)$-module with a $U\left(L_{-}\right)$-basis of weight vectors

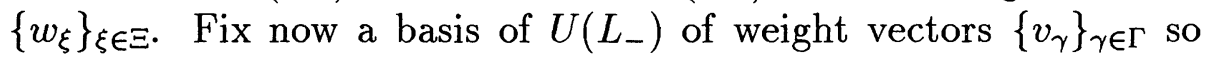
that $B=\left\{v_{\gamma} w_{\xi} \mid(\gamma, \xi) \in \Gamma \times \Xi\right\}$ is a basis of $F$. Now the fact that $\operatorname{dim} F_{\mu}<\infty$ for all $\mu \in \mathfrak{H}^{*}$ and $P(F) \subset \lambda-Q_{+}(\mathfrak{m})$ implies that one can enumerate the basis $B$ by negative integers, say $\left\{x_{i}\right\}_{i \in \mathbb{Z}_{<0}}$, so that

(1) each $x_{i}$ is a weight vector of the form $v_{\gamma} w_{\xi}$ and

(2) if $x_{i}$ has weight $\lambda_{i}$ and $\lambda_{i}>\lambda_{j}$, then $i>j$.

From now on we will assume that $F$ is an $\hat{\mathfrak{m}}$-subquotient of $M_{\mathfrak{m}}(\lambda)$ which has a basis $\left\{x_{i}\right\}$ that satisfies (1) and (2) above and we order this basis by $x_{i}<x_{j}$, if $i<j$. Note that $M_{\mathfrak{m}}(\lambda)$ also has a basis $\left\{x_{i}\right\}$ that satisfies (1) and (2).

Now suppose that $z \in \mathfrak{h}^{X}$ and $h \in \mathfrak{h}_{X}$ are both nonzero. Let $I$ be a finite subset of $\mathbb{Z}_{<0}$ and $\left\{m_{s}\right\}_{s \in S}$ a finite set of distinct integers. Let $e \ll 0$ and $(j, r) \in I \times S$. If one writes $x_{j}=v_{\gamma} w_{\xi}$ and commutes $h\left(e+m_{r}\right)$ past $v_{\gamma}$ (if $e \ll 0$ then no central terms appear) then one can rewrite $h\left(e+m_{r}\right) x_{j}$ in the form

$$
\sum_{\xi^{\prime}} a_{\xi^{\prime}} v_{\gamma} w_{\xi^{\prime}}
$$

where $a_{\xi^{\prime}} \in \mathbb{C}$. Then for $e \ll 0$ and $(i, s) \in I \times S$, Remark 3.2 and (1) above imply

$$
\begin{gathered}
\text { (3) } z\left(e+m_{s}\right) x_{i} \notin \operatorname{Span}\left\{z\left(e+m_{r}\right) x_{j}, h\left(e+m_{r}\right) x_{j} \mid\right. \\
(j, r) \neq(i, s),(j, r) \in I \times S\} .
\end{gathered}
$$


This is a key observation that we will use in the proof of

Proposition 4.5. Suppose $\lambda(c) \neq 0$ and $F$ is a subquotient of $M_{\mathfrak{m}}(\lambda)$ having a basis $\left\{x_{j}\right\}$ satisfying 4.4.(1) and (2). If $\beta \in$ $Q_{+} \backslash Q_{+}(\mathfrak{m})$ and $\operatorname{rad} U(F)_{\lambda-\beta} \neq 0$, then there exists $\alpha \in \dot{Q}_{+} \backslash \dot{Q}_{+}(\dot{\mathfrak{m}})$ and $p \in \mathbb{Z}$ such that $\operatorname{rad} U(F)_{\lambda-\beta+\alpha+p \delta} \neq 0$. More precisely if $0 \neq$ $u \in \operatorname{rad} U(F)_{\lambda-\beta} \neq 0$ for some $\beta \in Q_{+} \backslash Q_{+}(\mathfrak{m})$, then there exists $\alpha \in \dot{Q}_{+} \backslash \dot{Q}_{+}(\dot{\mathfrak{m}}), p \in \mathbb{Z}$, and $Y \in\left(U\left(\mathfrak{n}_{+}\right) \mathfrak{n}_{+}\right)_{\alpha+p \delta}$ such that $Y u \neq 0$.

Proof. $U\left(\mathfrak{u}_{-}\right)$has a basis $\left\{y_{\mathbf{i}, \mathbf{m}, \mathbf{p}}\right\}$ as in section three so that $\left\{y_{\mathbf{i}, \mathbf{m}, \mathbf{p}} x_{j}\right\}$ forms a basis of $U(F)$ that is lexicographically ordered

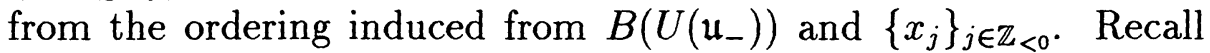
that $U\left(\mathfrak{u}_{-}\right)$is filtered by degree (see $\left.\S 3\right)$ and thus $U(F) \cong U\left(\mathfrak{u}_{-}\right) \otimes F$ is also filtered by degree in $U\left(\mathfrak{u}_{-}\right)$.

Let now $0 \neq u \in U(F)_{\lambda-\beta}$ with $\beta \in Q_{+} \backslash Q_{+}(\mathfrak{m})$. We have where $u_{\mathbf{i}, \mathbf{m}, \mathbf{p}}^{j} \in \mathbb{C}$. Let $y_{\mathbf{a}, \mathbf{b}, \mathbf{c}} x_{d}$ be a basis vector that is maximal among all terms with nonzero coefficients in $u$ above. Set $\bar{y}_{\max }=y_{\mathbf{a}, \mathbf{b}, \mathbf{c}}$ and $\bar{x}_{\text {max }}=x_{d}$. Certainly $\beta \in Q_{+} \backslash Q_{+}(\mathfrak{m})$ implies $y_{\mathbf{a}, \mathbf{b}, \mathbf{c}} \neq 1$, so $\operatorname{deg} \bar{y}_{\max }>0$.

Case 1. $J_{-}(z, \bar{y}) \neq \emptyset$ for some $z \in B\left(\dot{\mathfrak{u}}_{+}\right) \cup B\left(\dot{\mathfrak{m}}_{+}\right)$and some $\bar{y}$ with maximal degree occurring in the decomposition of $u$ above. Fix one such $z \in B\left(\dot{\mathfrak{u}}_{+}\right) \cup B\left(\dot{\mathfrak{m}}_{+}\right)$. Set

$$
\operatorname{Max}=\quad\left\{\bar{y}=y_{i_{1}}\left(m_{1}\right)^{p_{1}} \cdots y_{i_{r}}\left(m_{r}\right)^{p_{r}} \mid\right.
$$

$\bar{y}$ has maximal degree in $u$ with $\left.J_{-}(z, \bar{y}) \neq \emptyset\right\}$.

Then Max is not empty by hypothesis. Among all monomials $\bar{y} \in$ Max consider their factors $y_{i_{j}}$ and let $y_{a_{k}^{\prime}}$ be one in a root space $\dot{\mathfrak{g}}_{\alpha_{a_{k}^{\prime}}}$ where $\alpha_{a_{k}^{\prime}}$ is a minimal root among all such factors. In other words $\alpha_{a_{k}^{\prime}}$ is a minimal element in

$\left\{\alpha_{i_{j}} \mid y_{i_{j}} \in \dot{\mathfrak{g}}_{\alpha_{i_{j}}}\right.$, and $y_{i_{j}}$ occurs as a factor in some $\bar{y}$ with $\left.\bar{y} \in \operatorname{Max}\right\}$.

Let $\bar{y}_{\text {min }}=y_{\mathbf{a}^{\prime}, \mathbf{b}^{\prime}, \mathbf{c}^{\prime}} \in$ Max be a monomial that contains the factor $y_{a_{k}^{\prime}}$ and define $J_{\min }=J_{\min }(z)=J_{-}\left(z, \bar{y}_{\min }\right)$ and $J_{\min }^{c}=J^{c}\left(z, \bar{y}_{\min }\right)$. Let $j$ be an index such that $u_{\mathbf{a}^{\prime} \mathbf{b}^{\prime} \mathbf{c}^{\prime}}^{j} \neq 0$ and set $\bar{x}_{\min }=x_{j}$. We will now reduce to the case that all monomials with maximal degree have only factors $y_{i}$, lying in a root space $\dot{\mathfrak{g}}_{\alpha_{i_{1}}}$ with $\alpha_{i_{1}}$ simple. 


\section{By Lemma 4.3}

$z(e) \bar{y}_{\min } \bar{x}_{\min } \equiv \sum_{j \in J_{\min }} c_{j}\left[z, y_{a_{\jmath}^{\prime}}\right]\left(e+b_{j}^{\prime}\right) \bar{y}_{\min }^{\hat{j}} \bar{x}_{\min } \quad \bmod U\left(\mathfrak{u}_{-}\right)_{\left(c^{\prime}-1\right)} \otimes F$

where $c^{\prime}=\operatorname{deg} \bar{y}_{\text {min }}$ and either $e \ll 0$ and $z \in B\left(\dot{\mathfrak{u}}_{+}\right)$or $e \gg 0$ and $z \in B\left(\dot{\mathfrak{m}}_{+}\right)$. Let $S_{1}$ be the summation above. By Remark 3.2 the fact that $J_{\min }(z) \neq \emptyset$ and $e \ll 0$ or $e \gg 0$, implies $S_{1} \neq 0$. If $\bar{y}=\bar{y}_{\mathbf{i}, \mathbf{m}, \mathbf{p}}, \bar{x}=x_{j}$ is a different monomial occurring in $u$ then

$$
z(e) \bar{y} \bar{x} \equiv \sum_{j \in J_{-}} p_{j}\left[z, y_{i_{3}}\right]\left(e+m_{j}\right) \bar{y}^{\hat{j}} \bar{x} \quad \bmod U\left(\mathfrak{u}_{-}\right)_{(p-1)} \otimes F
$$

where $p=\operatorname{deg} \bar{y}$. Since $\bar{y}_{\min } \bar{x}_{\min }$ has maximal degree for the monomials in $u$ we have $p \leq c^{\prime}$. Let now $T_{1}$ be the summation above. In order for $T_{1} \in \operatorname{LinSpan}\left(S_{1}\right)+\operatorname{Span}\left(U\left(\mathfrak{u}_{-}\right)_{(p-1)} \otimes F\right)$ one must have $p=c^{\prime}$ and by Remark 3.2 , one also has $\mathbb{C}\left[z, y_{i_{\jmath}}\right]=\mathbb{C}\left[z, y_{a_{l}^{\prime}}\right]$, $m_{j}=b_{l}^{\prime}, \bar{y}_{\min }^{\hat{j}}=\bar{y}^{\hat{l}}$ and $\bar{x}=\bar{x}_{\text {min }}$ as $e \ll 0$ or $e \gg 0$. Consequently $\bar{y}_{\min }=\bar{y}$ and $\bar{x}=\bar{x}_{\text {min }}$ which is a contradiction. Hence $T_{1} \notin \operatorname{LinSpan}\left(S_{1}\right)+\operatorname{Span}\left(U\left(\mathfrak{u}_{-}\right)_{(p-1)} \otimes F\right)$.

Now our element $z(e) u$ has the same degree $c^{\prime}$ as $u$ and $z(e)$ sends each monomial of maximum degree to a monomial that is of higher weight with respect to the finite root lattice $\dot{Q}$. Since there are only finitely many nonzero weight spaces in $\dot{\mathfrak{g}}^{\otimes^{c^{\prime}}}$, after applying case (1) to $u$ finitely many times we arrive at

Case 2. $J_{-}(z, \bar{y})=\emptyset$ for all $z \in B\left(\dot{\mathfrak{u}}_{+}\right) \cup B\left(\dot{\mathfrak{m}}_{+}\right)$and all $\bar{y}$ with maximal degree occurring in the decomposition of $u$ above: This implies that if $\bar{y}=y_{\mathbf{i}, \mathbf{m}, \mathbf{p}}$ is a monomial in $u$ of the same degree as $\bar{y}_{\max }$ (recall $\bar{y}_{\max } \bar{x}_{\max }$ is the maximal element occurring in the decomposition of $u$ ), then the summations with indices in $J_{-}(z, \bar{y})$ in the formula 4.3 vanish. By Lemma 4.2 above we know that for all such maximal degree monomials $y_{\mathbf{i}, \mathbf{m}, \mathbf{p}}=y_{i_{1}}\left(m_{1}\right)^{p_{1}} \cdots y_{i_{r}}\left(m_{r}\right)^{p_{r}}$, and for all the factors $y_{i_{j}} \in \mathfrak{g}_{-\alpha_{2}}, \alpha_{i_{j}} \in \Delta\left(\mathfrak{u}_{+}\right)$are simple roots. In particular this holds for $\bar{y}_{\max }=y_{a_{1}}\left(b_{1}\right)^{c_{1}} \cdots y_{a_{s}}\left(b_{s}\right)^{c_{s}}$. Then for 
$e \ll 0$ and $1 \leq l \leq s$, one has by Lemma 4.3

$$
\begin{aligned}
& y_{-a_{l}}(e) \bar{y}_{\max } \bar{x}_{\max } \equiv \sum_{\substack{1 \leq j \leq s \\
a_{j}=a_{l}}}-c_{j}\left(c_{j}-1\right) y_{a_{j}}\left(e+2 b_{j}\right) \bar{y}_{\max }^{\hat{j} \hat{j}} \bar{x}_{\max } \\
& +\sum_{\substack{1 \leq j \leq s-1 \\
a_{j}=a_{l}}} \sum_{\substack{\xi=j+1 \\
s}}^{s} c_{j} c_{\xi} \alpha_{a_{\xi}}\left(h_{a_{l}}\right) y_{a_{\xi}}\left(b_{j}+b_{\xi}+e\right) \bar{y}_{\max }^{\hat{j} \hat{\xi}} \bar{x}_{\max } \\
& +\sum_{\substack{1 \leq j \leq s \\
a_{j}=a_{l}}} c_{j} \bar{y}_{\max }^{\hat{j}} h_{a_{l}}\left(b_{j}+e\right) \bar{x}_{\max } \quad \bmod U\left(\mathfrak{u}_{-}\right)_{(c-2)} \otimes F .
\end{aligned}
$$

Let $S_{1}, S_{2}$, and $S_{3}$ denote the first, second, and third summation respectively above. We will now consider, for a fixed $l, 1 \leq l \leq s$, the summand

$$
w=\bar{y}_{\max }^{\hat{l}} h_{a_{l}}\left(e+b_{l}\right) \bar{x}_{\max }
$$

in the summation $S_{3}$. Now $h_{a_{l}}=z+h$ where $0 \neq z \in \mathfrak{h}^{X}$ and $h \in \mathfrak{h}_{X}$. For $e \ll 0$ the element $w$ is thus nonzero by 4.4.(3). In addition observation 4.4.(3) also implies that $w$ is not in

$$
\operatorname{LinSpan}\left(\sum_{\substack{1 \leq j \leq s, j \neq l \\ a_{j}=a_{l}}} c_{j} \bar{y}_{\max }^{\hat{j}} h_{a_{l}}\left(b_{j}+e\right) \bar{x}_{\max }\right)+\operatorname{Span}\left(U\left(\mathfrak{u}_{-}\right)_{(c-2)} \otimes F\right)
$$

Moreover $w \notin \operatorname{LinSpan}\left(S_{1}\right)+\operatorname{LinSpan}\left(S_{2}\right)+\operatorname{Span}\left(U\left(\mathfrak{u}_{-}\right)_{(c-2)} \otimes F\right)$ as $S_{1}$ and $S_{2}$ have factors $y_{a_{j}}\left(2 b_{j}+e\right)$ or $y_{a_{\xi}}\left(b_{\xi}+b_{j}+e\right)$ in them with $e \ll 0$ (use Remark 3.2).

On the other hand consider an index $(\mathbf{i}, \mathbf{m}, \mathbf{p}, j) \neq(\mathbf{a}, \mathbf{b}, \mathbf{c}, d)$ and set $\bar{y}=y_{\mathbf{i}, \mathbf{m}, \mathbf{p}}=y_{i_{1}}\left(m_{1}\right)^{p_{1}} \cdots y_{i_{r}}\left(m_{r}\right)^{p_{r}}, \bar{x}=x_{j}$. Then by Lemma 4.3 
we have for $e \ll 0$ (so that $J_{-}^{r}=\emptyset$ )

$$
\begin{aligned}
& y_{-a_{l}}(e) \bar{y} \bar{x} \equiv \sum_{j \in J_{-}} p_{j}\left[y_{-a_{l}}, y_{i_{j}}\right]\left(e+m_{j}\right) \bar{y}^{\hat{j}} \bar{x} \\
& +\sum_{j \in J^{c}} p_{j} \bar{y}^{\hat{j}}\left[y_{-a_{l}}, y_{i_{j}}\right]\left(e+m_{j}\right) \bar{x} \\
& +\sum_{j=1}^{r} \epsilon_{j}\left[y_{i_{j}},\left[y_{-a_{l}}, y_{i_{j}}\right]\right]\left(e+2 m_{j}\right) \bar{y}^{\hat{j} \hat{j}} \bar{x} \\
& +\sum_{j \in J_{-} \backslash\{1\}} \sum_{\xi=1}^{j-1} p_{j} p_{\xi}\left[\left[y_{-a_{l}}, y_{i_{j}}\right], y_{i_{\xi}}\right]\left(e+m_{j}+m_{\xi}\right) \bar{y}^{\hat{j} \hat{\xi}} \bar{x} \\
& +\sum_{j \in J^{c} \backslash\{r\}} \sum_{\xi=j+1}^{r} p_{j} p_{\xi}\left[\left[y_{-a_{l}}, y_{i_{\jmath}}\right], y_{i_{\xi}}\right] \\
& \cdot\left(e+m_{j}+m_{\xi}\right) \bar{y}^{\hat{j} \hat{\xi}} \bar{x} \bmod U\left(\mathfrak{u}_{-}\right)_{(p-2)} \otimes F
\end{aligned}
$$

where $p=\operatorname{deg} \bar{y}$ and $\epsilon_{j} \in \mathbb{Z}$. For $1 \leq i \leq 5$, let $T_{i}$ be the $i$ th summation above. Our goal is to show that none of the $w \notin$ $\sum_{i=1}^{5} \operatorname{LinSpan}\left(T_{i}\right)+\operatorname{Span}\left(U\left(\mathfrak{u}_{-}\right)_{(p-1)} \otimes F\right)$.

Suppose $p \leq c^{\prime}-1$, then $T_{2}, T_{3}, T_{4}, T_{5}$ all have degree $c^{\prime}-2$ or less so

$$
w \notin \sum_{i=2}^{5} \operatorname{LinSpan}\left(T_{i}\right)+\operatorname{Span}\left(U\left(\mathfrak{u}_{-}\right)_{(p-1)} \otimes F\right) .
$$

But $w \notin \operatorname{LinSpan}\left(T_{1}\right)+\operatorname{Span}\left(U\left(\mathfrak{u}_{-}\right)_{(p-1)} \otimes F\right)$ as $e \ll 0$. Thus

$$
w \notin \sum_{i=1}^{5} \operatorname{LinSpan}\left(T_{i}\right)+\operatorname{Span}\left(U\left(\mathfrak{u}_{-}\right)_{(p-1)} \otimes F\right) .
$$

Suppose now $p=c^{\prime}$. Here $y_{\mathbf{i}, \mathbf{m}, \mathbf{p}}$ has maximal degree so each factor $y_{i}$ of $y_{\mathbf{i}, \mathbf{m}, \mathbf{p}}$ must come from a root space of a simple root $\alpha_{i_{j}} . T_{1}=$ $T_{4}=0$ by the hypothesis of Case 2 and thus $w \notin \sum_{i=1,4} \operatorname{LinSpan}\left(T_{i}\right)+$ $\operatorname{Span}\left(U\left(\mathfrak{u}_{-}\right)_{(p-1)} \otimes F\right)$. Since $\alpha_{i_{j}}$ is simple and so is $\alpha_{a_{l}}$ we have that

$$
\left[y_{-a_{l}}, y_{i_{j}}\right]=h_{a_{l}} \text { or } 0
$$

depending on whether $i_{j}=a_{l}$ or not. Thus

$$
\left[y_{i_{\xi}},\left[y_{-a_{l}}, y_{i_{j}}\right]\right]\left(e+m_{j}+m_{\xi}\right)=\alpha_{i_{\xi}}\left(h_{a_{l}}\right) y_{i_{\xi}}\left(e+m_{j}+m_{\xi}\right) \text { or } 0,
$$


accordingly. Hence for $e \ll 0$ we have that

$$
w \notin \sum_{i=1,3,4,5} \operatorname{LinSpan}\left(T_{i}\right)+\operatorname{Span}\left(U\left(\mathfrak{u}_{-}\right)_{(p-1)} \otimes F\right)
$$

This leaves us to study $T_{2}$. Now in order for

$$
w \in \sum_{i=1}^{5} \operatorname{LinSpan}\left(T_{i}\right)+\operatorname{Span}\left(U\left(\mathfrak{u}_{-}\right)_{(p-1)} \otimes F\right)
$$

one must have by Remark 3.2 that

$$
\bar{y}^{\hat{j}}=\bar{y}_{\max }^{\hat{l}} \quad \text { and } \quad\left[y_{-a_{l}}, y_{i_{j}}\right]\left(e+m_{j}\right) \bar{x}=h_{a_{l}}\left(e+b_{l}\right) \bar{x}_{\max }
$$

Using the observation 4.4.(3) one has that $\bar{x}_{\max }=\bar{x}$ and $m_{j}=b_{l}$. Thus $y_{i_{j}}\left(m_{j}\right)=y_{a_{l}}\left(b_{l}\right)$ so that $\bar{y}=\bar{y}_{\max }$. But this contradicts the assumption $(\mathbf{i}, \mathbf{m}, \mathbf{p}, j) \neq(\mathbf{a}, \mathbf{b}, \mathbf{c}, d)$.

THEOREM 4.6. If $\lambda(c) \neq 0$ then $U(F)$ is irreducible if and only if $F$ is irreducible.

Proof. Suppose $F$ is a reducible subquotient of $M_{\mathfrak{m}}(\lambda)$ i.e. there exists a proper $\hat{\mathfrak{m}}$-module $N$ of $F$. Then $\mathfrak{u}_{+} \cdot N=0$ when we view $N \subset U(F)$ and $N_{\mu} \neq 0$ for some $\mu \in \lambda-Q_{+}(\hat{\mathfrak{m}})$ so that $N^{\prime}:=$ $U(\mathfrak{g}) N=U\left(\mathfrak{u}_{-}\right) N \subsetneq U(F)$ is a proper $\mathfrak{g}$-submodule. Hence $U(F)$ is reducible.

For the converse we assume that $\lambda(c) \neq 0$ and $F$ is irreducible. First we claim that $\operatorname{rad}(U(F))_{\lambda-\beta}=0$ for $\beta \in Q_{+}(\mathfrak{m})$. This is because $\left(\operatorname{rad}_{\hat{\mathfrak{g}}} U(F)\right)_{\lambda-\beta} \subset F_{\lambda-\beta}$ for all irreducible subquotients $F$ of $M_{\mathfrak{m}}(\lambda)$ and $\beta \in Q_{+}(\mathfrak{m})$. Thus if $F$ is irreducible and $\operatorname{rad}_{\hat{\mathfrak{g}}} U(F)_{\lambda-\beta} \neq$ 0 for some $\beta$ then $\operatorname{rad}_{\hat{\mathfrak{g}}} U(F)$ contains a generator of $F$ and hence $\operatorname{rad}_{\hat{\mathfrak{g}}} U(F)=U(F)$. Clearly this is nonsense. Now if $\operatorname{rad}(U(F))_{\lambda-\beta} \neq$ 0 for some $\beta \in Q_{+}$we could use Proposition 4.5 inductively to obtain a nonzero element in $\operatorname{rad}(U(F))_{\lambda-\beta^{\prime}}$ for some $\beta^{\prime} \in Q_{+}(\mathfrak{m})$ which leads again to a contradiction. Finally from Proposition 1.5 we see that $\operatorname{rad} U(F)=0$ i.e $U(F)$ is irreducible. 


\section{Composition Series and Embeddings of Verma mod-} ules for $\lambda(c) \neq 0$. In this section we restrict ourselves to the case that $\lambda(c) \neq 0$.

Let $\mu \in \mathfrak{H}^{*}$ and $M$ be a module in $\mathcal{O}^{X}$. As in section two, a sequence $0=M_{0} \subset M_{1} \subset \cdots \subset M_{k}=M$ of modules in $\mathcal{O}^{X}$ and a subset $I \subset\{1, \ldots, k\}$ such that

(1) if $i \in I$, then $M_{i} / M_{i-1} \cong L^{X}\left(\mu_{i}\right)$ for some $\mu_{i} \geq \mu$ (partially ordered with respect to $\left.Q_{+}\right)$,

(2) if $i \notin I$ then $\left(M_{i} / M_{i-1}\right)_{\lambda}=(0)$ for all $\lambda_{i} \geq \mu$

is a local composition series at $\mu$. If $\mu_{i} \geq \mu$, then we let $\left[M: L\left(\mu_{i}\right)\right]$ be the number of times that $L(\mu)$ appears in (1) above. Of course this definition may depend on the sequence $M_{1} \subset \cdots \subset M_{k}=$ $M$ above. Consider a countable direct sum of irreducible modules $\oplus_{i \in \mathbb{Z}} L(\mu)$. This module is in the category $\mathcal{O}^{X}$ but it does not have a local composition series at $\mu$. So this notion is not well defined for all modules in $\mathcal{O}^{X}$. On the other hand, for $M=M(\lambda)$ with $\lambda(c) \neq 0$ this number is well defined (see 5.2 below).

We will now essentially follow the argument of [4] Lemma 9.

For a $\hat{\mathfrak{g}}$-submodule $N$ of $M(\lambda)$ let $[N]:=N \cap M_{\mathfrak{m}}(\lambda)$.

LEMMA 5.1. Every nonzero submodule $N$ of $M(\lambda)$ is generated by $[N]$ and $[N] \neq 0$.

Proof. We may assume that $N$ is a proper submodule of $M(\lambda)$. If $0 \neq u \in N$, then by Proposition 4.5 there exists a $Y \in U(\hat{\mathfrak{g}})$ such that $0 \neq Y u \in \operatorname{rad} M_{\mathfrak{m}}(\lambda)$. Hence $[N] \neq 0$. Let $N^{\prime}$ be the $\hat{\mathfrak{g}}$ submodule of $N$ generated by $[N]$ i.e. $N^{\prime}=U\left(\mathfrak{u}_{-}\right)[N]$ and suppose that $N^{\prime} \neq N$. Then $N / N^{\prime} \subset M(\lambda) / U\left(\mathfrak{u}_{-}\right)[N] \cong U\left(M_{\mathfrak{m}}(\lambda) /[N]\right)$. Let

(1) $u=\sum y_{\mathbf{i}, \mathbf{m}, \mathbf{p}} x_{\mathbf{i}, \mathbf{m}, \mathbf{p}}$

where the $y_{\mathbf{i}, \mathbf{m}, \mathbf{p}}$ are basis vectors in $U\left(\mathfrak{u}_{-}\right)$as in section three and where the $x_{\mathbf{i}, \mathbf{m}, \mathbf{p}}$ are elements of $M_{\mathfrak{m}}(\lambda) /[N]$. If $u \neq 0$ in $N / N^{\prime}$ then $x_{\mathbf{i}, \mathbf{m}, \mathbf{p}} \neq 0$ for some index $(\mathbf{i}, \mathbf{m}, \mathbf{p})$ appearing in the decomposition of $u$. Let $\mu \in \mathfrak{h}^{*}$ be such that each such nonzero $x_{\mathbf{i}, \mathbf{m}, \mathbf{p}}$ is a linear combination of weight vectors in $M_{\mathfrak{m}}(\lambda) /[N]$ of weight greater than or equal to $\mu$. Now consider a local composition series for $M_{\mathfrak{m}}(\lambda) /[N]$ at $\mu$ : $0=M_{0} \subset M_{1} \subset \cdots \subset M_{k}=M_{\mathfrak{m}}(\lambda) /[N]$. Let $1 \leq d \leq k$ be 
maximal with respect to the property that some $x_{\mathbf{i}, \mathbf{m}, \mathbf{p}}$ has nonzero image $\bar{x}_{\mathbf{i}, \mathbf{m}, \mathbf{p}}$ in $M_{d} / M_{d-1}$. Let $x_{\mathbf{a}, \mathbf{b}, \mathbf{c}}$ be one such nonzero element in $M_{\mathfrak{m}}(\lambda) /[N]$ occurring in the decomposition (1). Then since $x_{\mathbf{a}, \mathbf{b}, \mathbf{c}}$ is a linear combination of elements of weight greater than or equal to $\mu$ we have $M_{d} / M_{d-1} \cong L\left(\mu^{\prime}\right)$ for some $\mu^{\prime} \geq \mu$. Moreover $u \in U\left(u_{-}\right) M_{d}$ by the maximality of $d$ and $u \notin U\left(\mathfrak{u}_{-}\right) M_{d-1}$ since $U\left(M_{\mathfrak{m}}(\lambda)\right) /[N]$ is a free $U\left(\mathfrak{u}_{-}\right)$-module (Remark 3.2). Hence $u$ has nonzero image $\bar{u}$ in the quotient $U\left(M_{d} / M_{d-1}\right) \cong U\left(\mathfrak{u}_{-}\right) \otimes M_{d} / M_{d-1}$. By Proposition 4.5, $U(\hat{\mathfrak{g}}) \bar{u} \cap U\left(M_{d} / M_{d-1}\right)_{\lambda-\beta} \neq 0$ for some $\beta \in Q_{+}(\mathfrak{m})$. But this implies that $(N /[N])_{\lambda-\beta}=\left(N / N^{\prime}\right)_{\lambda-\beta} \neq 0$ for some $\beta \in Q_{+}(\mathfrak{m})$ which contradicts the fact that $N_{\lambda-\beta}=[N]_{\lambda-\beta}$ for all $\beta \in Q_{+}(\mathfrak{m})$.

THEOREM 5.2. Let $\lambda, \mu \in \mathfrak{h}^{*}$. If $\lambda(c) \neq 0$ and $\mu(c) \neq 0$ then

$$
[M(\lambda): L(\mu)]=\left[M_{\mathfrak{k}}(\lambda): L_{\mathfrak{k}}(\mu)\right] \text {. }
$$

Proof. Lemma 2.2 and Theorem 4.6 proves that a local composition series exists for Verma modules $M(\lambda)$ with $\lambda$ satisfying $\lambda(c) \neq 0$ (in particular a local composition series for $M_{\mathfrak{m}}(\lambda)$ induces a local composition series for $M(\lambda)$.) From the previous lemma we see that every irreducible subquotient of $M(\lambda)$ is isomorphic to $U(F)$ for some irreducible $\hat{\mathfrak{m}}$-subquotient $F$ of $M_{\mathfrak{m}}(\lambda)$ and any local composition series for $M(\lambda)$ comes from a local composition series of $M_{\mathfrak{m}}(\lambda)$. Consequently the multiplicity $[M(\lambda): L(\mu)]$ is independent of local composition series used and Proposition 2.3 gives us the multiplicity of irreducible subquotients in such a series.

Corollary 5.3. Let $\lambda, \mu \in \mathfrak{h}^{*}$. If $\lambda(c) \neq 0$ and $\mu(c) \neq 0$, then the map $\phi \mapsto \bar{\phi}:=\left.\phi\right|_{M_{\mathrm{k}}(\lambda)}$ defines an isomorphism of vector spaces (1) $\quad: \operatorname{Hom}_{\hat{\mathfrak{g}}}(M(\lambda), M(\mu)) \rightarrow \operatorname{Hom}_{\hat{\mathfrak{k}}}\left(M_{\mathfrak{k}}(\lambda), M_{\mathfrak{k}}(\mu)\right)$.

Proof. Since $\phi$ is an $\hat{\mathfrak{g}}$-module map, the map $\check{\phi}:=\left.\phi\right|_{M_{\mathfrak{m}}(\lambda)}$ : $M_{\mathfrak{m}}(\lambda) \rightarrow M(\mu)$ is an $\hat{\mathfrak{m}}$-module homomorphism. Now if $v_{+}$denotes the highest weight vector of $M(\lambda)$, then $\phi\left(v_{+}\right) \in \operatorname{Vac}_{\mathfrak{n}_{+}} M(\mu)$ so by Proposition 4.5, $\phi\left(v_{+}\right) \in M_{\mathfrak{m}}(\mu)$. Thus $\check{\phi}$ has image in $M_{\mathfrak{m}}(\mu)$ and we have $\check{\phi}: M_{\mathfrak{m}}(\lambda) \rightarrow M_{\mathfrak{m}}(\mu)$. By Proposition $4.5 \check{\phi}$ is not zero if $\phi$ is not zero. Consequently the map

$: \operatorname{Hom}_{\hat{\mathfrak{g}}}(M(\lambda), M(\mu)) \rightarrow \operatorname{Hom}_{\hat{\mathfrak{m}}}\left(M_{\mathfrak{m}}(\lambda), M_{\mathfrak{m}}(\mu)\right)$. 
is injective. Using the universal mapping property of Verma modules the reader can check that ${ }^{-}$is also surjective. This map combined with the one in Proposition 2.3 gives the isomorphism (1) above.

\section{Index of Notation}

$1.1 \mathbb{Z}_{R c}, \hat{\mathfrak{g}}, \mathfrak{n}_{-}, \mathfrak{h}, \dot{\mathfrak{n}}_{+}, \dot{\Delta}, \Pi, \dot{\Delta}_{+}, \pi, L(\dot{\mathfrak{g}}), c, d, X, \dot{\Delta}^{X}, \dot{\Delta}_{ \pm}^{X}, \mathfrak{H}$, $\mathfrak{h}_{X}, \mathfrak{h}^{X}, \dot{\mathfrak{m}}_{ \pm}^{X}, \dot{\mathfrak{m}}^{X}, \mathfrak{m}_{ \pm}^{X}, \hat{\mathfrak{m}}^{X}, \dot{\mathfrak{u}}_{ \pm}^{X}, \mathfrak{u}_{ \pm}^{X}, \mathfrak{k}_{ \pm}^{X}, \hat{\mathfrak{k}}^{X}, \mathfrak{n}_{ \pm}^{X}, \mathfrak{b}_{ \pm}^{X}, \mathfrak{p}_{ \pm}^{X}$

$1.2 \Delta_{+}^{X}, \Delta_{ \pm}\left(\hat{\mathfrak{m}}^{X}\right), \Delta_{ \pm}\left(\mathfrak{u}^{X}\right), Q_{+}^{X}, Q_{+}(\mathfrak{m}), \lambda<^{X} \mu, \dot{Q}$,

$1.3 V_{\lambda}, P(V), D_{X}(\lambda), \mathcal{O}^{X}=\mathcal{O}^{X}(\hat{\mathfrak{g}}), \mathcal{O}^{X}(\hat{\mathfrak{m}}), \mathcal{O}^{X}(\hat{\mathfrak{k}}), M^{(n)}, \operatorname{rad} P$,

$1.4 U(L), M^{X}(\lambda), M_{\mathfrak{m}}^{X}(\lambda), M_{\mathfrak{k}}^{X}(\lambda), L_{\mathfrak{m}}^{X}(\lambda), L_{\mathfrak{k}}^{X}(\lambda), U(N)$,

$1.5 L(\lambda)$

$2.0\left(\mathfrak{h}, \hat{\mathfrak{m}}_{+}, Q_{+}(\mathfrak{m}),\left.\sigma\right|_{\hat{\mathfrak{m}}}\right),\left(\mathfrak{h}, \hat{\mathfrak{k}}_{+}, Q_{+}(\mathfrak{m}),\left.\sigma\right|_{\hat{\mathfrak{k}}}\right), \mathcal{O}(\mathfrak{g}, T), M_{T}(\lambda), L_{T}(\lambda)$, $\mathfrak{C}_{k}, \operatorname{Vac} V, L=L^{X}$,

$2.1 M(k)$,

$2.2\left[M: L_{T}(\mu)\right]$

$3.0 x(m)=x \otimes t^{m}, \bar{y}^{\hat{\xi} \hat{j}}, B\left(U\left(\mathfrak{u}_{-}\right)\right), U\left(\mathfrak{u}_{-}\right)_{(p)}, B\left(\mathfrak{u}_{-}\right), B\left(\mathfrak{u}_{+}\right)$,

$4.3 \bar{y}_{\max }, \bar{x}_{\max }$,

$4.4 J_{+}(z, \bar{y}), J_{0}(z, \bar{y}), J_{-}(z, \bar{y}), \epsilon_{j}$,

$4.5 J_{\max }=. J_{\max }(z)=J_{-}\left(z, \bar{y}_{\max }\right)$,

$5.0[N],[M: L(\mu)]$ 


\section{Bibliography}

[1] V.Chari,Integrable Representations of Affine Lie Algebras,Invent. Math., 85 (1986), p. 317-335.

[2] V. Chari, A. Pressley, A New Family of Irreducible, Integrable Modules for Affine Lie Algebras, Math. Ann., 277 (1987),p. 543-652.

[3] V. Deodhar, O. Gabber, V. Kac,Structure of Some Categories of Representations of Infinite Dimensional Lie Algebras, Adv. in Math. , 45(1982), p.92-116.

[4] V. Futorny, On imaginary Verma modules over the affine $A_{1}^{(1)}$, preprint from Univ. of Oslo,1991.

[5] I. Frenkel, J. Lepowski, A. Meurman, Vertex Operator Algebras and the Monster,Academic Press, 1989.

[6] P. Hilton, U. Stammbach, A Course in Homological Algebra, SpringerVerlag, 1970.

[7] H. Jakobsen, V. Kac, A new class of unitarizable highest weight representations of infinite dimensional Lie algebras, Lect. Notes in Physics, Springer-Verlag, 226(1985), p. 1-20.

[8] V. Kac,Infinite dimensional Lie algebras, Cambridge Univ. Press.,1985.

[9] R. Moody, A. Pianzola, Lie algebras with triangular decomposition, J. Wiley, 1995.

[10] D. Peterson, V. Kac,Infinite flag varieties and conjugacy theorems, Proc. Natl. Acad. Sci. USA, 80(1983), p. 1778-1782.

Received February 3, 1992, revised September 15, 1992 and accepted for publication January 18, 1993. Supported in part by the University of Montana Research Grant Program.

Department of Mathematical Sciences

UNIVERSITY OF MONTANA

Missoula MT. 59812-1032

E-mail address: ma-blc@selway.umt.edu 


\section{CONTENTS}

S. Abdulali, Conjugates of strongly equivariant maps .............. 207

C. Adams, Dehn filling hyperbolic 3-manifolds ...................... 217

S. Berman and B. Cox, Enveloping algebras and representations of toroidal Lie algebras.......................................... 239

B. Cox, Verma modules induced from nonstandard Borel subalgebras . 269

C. H. FitzGerald and C. R. Thomas, Some bounds on convex mappings in several complex variables..., ...................... 295

P. H. Loi, On the derived towers of certain inclusions of type $I I I_{\lambda}$ fac-

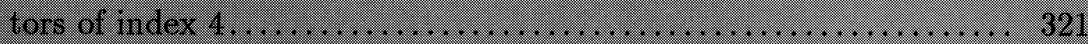

F. Morgan, Soap bubbles in $\mathbb{R}^{2}$ and in surfaces..................... 347

M. Shiffman, Partially measurable sets in measur spaces ............. 363 


\section{PACIFIC JOURNAL OF MATHEMATICS}

Volume $165 \quad$ No. $2 \quad$ October 1994

Conjugates of strongly equivariant maps

207

SALMAN ABDULALI

Dehn filling hyperbolic 3-manifolds

217

COLIN C. ADAMS

Enveloping algebras and representations of toroidal Lie algebras

239

STEPHEN BERMAN and BEN COX

Verma modules induced from nonstandard Borel subalgebras

269

BEN COX

Some bounds on convex mappings in several complex variables

295

CARL HANSON FitzGERALD and CAROLYN R. THOMAS

On the derived towers of certain inclusions of type $I I I_{\lambda}$ factors of

321 index 4

PHAN HUNG LOI

Soap bubbles in $\mathbb{R}^{2}$ and in surfaces

347

FRANK MORGAN

Partially measurable sets in measure spaces

MAX SHIFFMAN 